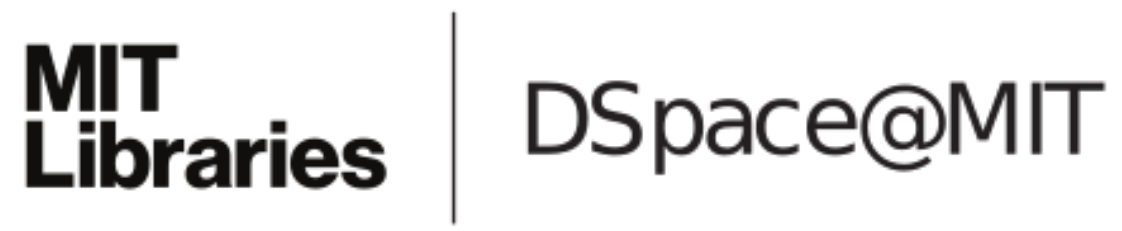

\author{
MIT Open Access Articles
}

\author{
COMMON PATTERNS IN THE EVOLUTION \\ BETWEEN THE LUMINOUS NEUTRON STAR \\ LOW-MASS X-RAY BINARY SUBCLASSES
}

The MIT Faculty has made this article openly available. Please share how this access benefits you. Your story matters.

Citation: Fridriksson, Joel K., Jeroen Homan, and Ronald A. Remillard. “COMMON PATTERNS IN THE EVOLUTION BETWEEN THE LUMINOUS NEUTRON STAR LOW-MASS X-RAY BINARY SUBCLASSES." The Astrophysical Journal 809, no. 1 (August 10, 2015): 52. () 2015 The American Astronomical Society

As Published: http://dx.doi.org/10.1088/0004-637x/809/1/52

Publisher: IOP Publishing

Persistent URL: http://hdl.handle.net/1721.1/99904

Version: Final published version: final published article, as it appeared in a journal, conference proceedings, or other formally published context

Terms of Use: Article is made available in accordance with the publisher's policy and may be subject to US copyright law. Please refer to the publisher's site for terms of use. 


\title{
COMMON PATTERNS IN THE EVOLUTION BETWEEN THE LUMINOUS NEUTRON STAR LOW-MASS X-RAY BINARY SUBCLASSES
}

\author{
Joel K. Fridriksson ${ }^{1,2}$, Jeroen Homan ${ }^{2,3}$, and Ronald A. Remillard ${ }^{2}$ \\ ${ }^{1}$ Anton Pannekoek Institute for Astronomy, University of Amsterdam, Science Park 904, 1098 XH Amsterdam, The Netherlands; J.K.Fridriksson@uva.nl \\ ${ }^{2}$ Kavli Institute for Astrophysics and Space Research, Massachusetts Institute of Technology, 77 Massachusetts Avenue, Cambridge, MA 02139, USA \\ ${ }^{3}$ SRON, Netherlands Institute for Space Research, Sorbonnelaan 2, 3584 CA Utrecht, The Netherlands \\ Received 2014 September 12; accepted 2015 March 29; published 2015 August 10
}

\begin{abstract}
The X-ray transient XTE J1701-462 was the first source observed to evolve through all known subclasses of lowmagnetic-field neutron star low-mass X-ray binaries (NS-LMXBs), as a result of large changes in its mass accretion rate. To investigate to what extent similar evolution is seen in other NS-LMXBs we have performed a detailed study of the color-color and hardness-intensity diagrams (CDs and HIDs) of Cyg X-2, Cir X-1, and GX 13+1-three luminous X-ray binaries, containing weakly magnetized neutron stars, known to exhibit strong secular changes in their CD/HID tracks. Using the full set of Rossi X-ray Timing Explorer Proportional Counter Array data collected for the sources over the 16 year duration of the mission, we show that Cyg X-2 and Cir X-1 display CD/HID evolution with close similarities to XTE J1701-462. Although GX 13+1 shows behavior that is in some ways unique, it also exhibits similarities to XTE J1701-462, and we conclude that its overall CD/HID properties strongly indicate that it should be classified as a $\mathrm{Z}$ source, rather than as an atoll source. We conjecture that the secular evolution of Cyg X-2, Cir X-1, and GX 13+1-illustrated by sequences of CD/HID tracks we construct—arises from changes in the mass accretion rate. Our results strengthen previous suggestions that within single sources Cyg-like Z source behavior takes place at higher luminosities and mass accretion rates than Sco-like $\mathrm{Z}$ behavior, and lend support to the notion that the mass accretion rate is the primary physical parameter distinguishing the various NS-LMXB subclasses.
\end{abstract}

Key words: accretion, accretion disks - stars: neutron - X-rays: binaries -

X-rays: individual (Cir X-1, Cyg X-2, GX 13+1, XTE J1701-462)

\section{INTRODUCTION}

In neutron star low-mass X-ray binaries (NS-LMXBs), a neutron star accretes matter from a low-mass companion star. The vast majority of NS-LMXBs contains weakly magnetized neutron stars, and these systems are traditionally divided into two main subclasses based on their correlated spectral and timing properties: the $\mathrm{Z}$ sources and atoll sources (Hasinger \& van der Klis 1989). The names derive from the shapes of the tracks the sources trace out in the X-ray color-color and hardness-intensity diagrams (CDs and HIDs). The Z sources are the more luminous, with near-Eddington X-ray luminositities, whereas the atoll sources are thought to in general have $L_{\mathrm{X}} \lesssim 0.5 L_{\text {Edd }}$. Further divisions of these two main NS-LMXB types have also been made; in particular, the $\mathrm{Z}$ sources have been divided into the Cyg-like and Sco-like subtypes (Kuulkers et al. 1997 and references therein).

In addition to differences in X-ray luminosity, rapidvariability characteristics, and spectral properties (see, e.g., van der Klis 2006 and references therein), the NS-LMXB subclasses also differ with respect to behavior in the radio band (Migliari \& Fender 2006) and the rates and properties of type I X-ray bursts exhibited (Galloway et al. 2008). Understanding what physical factors underlie this variety in characteristics has been a long-standing problem in the study of X-ray binaries. It has long been clear that the mass accretion rate must play an important role (e.g., it seems evident that $\mathrm{Z}$ sources must in general accrete at a higher rate than atoll sources, given the former's significantly higher luminosity), but exactly what that role is, and where other physical parameters enter the picture, has been debated.

Another long-standing question concerns the physical nature of the motion of $\mathrm{Z}$ sources in the CD/HID. The $\mathrm{Z}$ sources trace out characteristic tracks in the CD and HID (consisting of the so-called horizontal, normal, and flaring branches) on typical timescales of hours to a few days. Initially, it was assumed that motion along the $\mathrm{Z}$ track was driven by changes in the mass accretion rate, with $\dot{M}$ increasing monotonically from the tip of the horizontal branch to the tip of the flaring branch (e.g., Hasinger et al. 1990), but later observations cast doubts on this, and other scenarios have been proposed (see, e.g., van der Klis 2001; Homan et al. 2002, 2007; Church et al. 2012). In addition to motion along the tracks, these sources show another type of motion, usually on longer timescales (days to weeks), where the tracks can shift, primarily in the HID. This has been referred to as secular motion/shifts/changes/variations. In most cases these shifts are small and do not lead to significant changes in the shapes of the tracks. However, a few sources have been known to exhibit much stronger secular changes where the tracks shift and change shape radically in both the $\mathrm{CD}$ and HID.

A breakthrough in our understanding of the Z/atoll phenomenology and the nature of secular evolution came with the transient NS-LMXB XTE J1701-462, which over the course of its 2006-2007 outburst evolved through all subclasses of low-magnetic-field NS-LMXBs-from a Cyg-like Z source at the highest luminosities to a Sco-like one, followed by a phase in the atoll source soft state (during which type I X-ray bursts were seen), and ending with a transition to the atoll source hard state before returning to quiescence (Lin et al. 2009b; Homan et al. 2010). Lin et al. (2009b) performed a detailed spectral analysis of the entire outburst, using data from the Rossi X-ray Timing Explorer $(R X T E)$, and their results indicate that changes in the mass accretion rate were responsible for the evolution of the source. 
Homan et al. (2010)-hereafter referred to as H10 - further argue that the observed behavior of the source implies that differences in the mass accretion rate can alone explain the existence of the various NS-LMXB subclasses, and that it is not necessary to invoke differences in other parameters, such as the magnetic field of the neutron star (Hasinger \& van der Klis 1989; Psaltis et al. 1995) or the inclination angle of the system (Kuulkers \& van der Klis 1995).

The main goal of this paper is to study to what extent (if any) evolution similar to that of XTE J1701-462 is observed in other sources. Comparisons of XTE J1701-462's evolution at the lowluminosity end with atoll sources have been made in Lin et al. (2009b), H10, and Muñoz-Darias et al. (2014), showing that there the source behaved similar to other low-luminosity (atoll) NS-LMXBs; in this paper we focus mainly on the highluminosity (Z source) portion of XTE J1701-462's evolution. To this end we performed a detailed analysis of the CDs and HIDs of three luminous neutron star X-ray binaries-Cyg X-2, Cir X-1, and GX 13+1-using all available RXTE Proportional Counter Array (PCA) data. Similar to XTE J1701-462, these sources are known to have shown strong secular evolution (e.g., Oosterbroek et al. 1995; Kuulkers et al. 1996; Wijnands et al. 1997; Shirey et al. 1999a; Schnerr et al. 2003). Since we are mainly interested in sources that cross subclass boundaries, we do not include in our study persistent $\mathrm{Z}$ sources that show only mild secular shifts and no significant changes in the shapes of their tracks. Although small subsets of the RXTE data sets for Cyg X-2, Cir X-1, and GX 13+1 have been studied in several papers - usually with an emphasis on spectroscopy and/or timing analysis - we are not aware of any papers presenting a comprehensive study of the secular evolution of any of these sources using a large amount of $R X T E$ data, with the exception of Schnerr et al. (2003), who studied a large set of observations of GX 13+1 made in 1998 (see discussion in Section 3.4). However, since then the amount of GX 13+1 data in the $R X T E$ archive has grown considerably. We also note that Shirey and collaborators studied CDs and HIDs of Cir X-1 using a number of observations made in 1996 and 1997 in a series of papers (Shirey et al. 1996, 1998, 1999a; see discussion in Section 3.3), but these observations represent only a small fraction of the currently available $R X T E$ data for this source. In addition to our analysis of Cyg X-2, Cir X-1, and GX 13+1 we also perform some reanalysis of XTE J1701-462 in order to ensure complete consistency with the analysis of the other three sources and to facilitate comparisons between them and XTE J1701-462. We note that although Cir X-1 may be a rare low-magnetic-field neutron star high-mass X-ray binary (as further discussed in Section 3.3) we will for simplicity in general refer to all four sources studied in this paper as NS-LMXBs.

The structure of the paper is as follows. In Section 2 we describe the general data analysis steps common to all four sources, but leave the description of analysis specific to each source to Section 3. In Section 3.1 we briefly describe our analysis and present our results for XTE J1701-462. This analysis largely follows that previously performed by $\mathrm{H} 10$ (and includes creating a sequence of CD/HID tracks that shows the secular evolution of the source); we therefore mostly refer to that paper for details. In this section we also tie this source's behavior to the various source states/branches generally seen in the CDs and HIDs of NS-LMXBs. In Sections 3.2-3.4 we describe our analysis and present our results for Cyg X-2, Cir X-1, and GX 13+1, respectively. For
Table 1

Total Exposure Times for Analyzed Sources

\begin{tabular}{lc}
\hline \hline Source & Exp. Time (Ms) \\
\hline XTE J1701-462 & 2.71 \\
Cyg X-2 & 2.28 \\
Cir X-1 & 2.57 \\
GX 13+1 & 0.58 \\
\hline
\end{tabular}

each source we construct a sequence of CD/HID tracks (analogous to the one for XTE J1701-462) that illustrates its secular evolution; we also give a brief background on each of these three sources. In Section 4 we discuss our results, and in Section 5 we give a summary of our results and conclusions.

\section{DATA ANALYSIS}

\subsection{Data Extraction}

We used the PCA data from all pointed RXTE observations of XTE J1701-462, Cyg X-2, Cir X-1, and GX 13+1 obtained during the lifetime of the mission. In Table 1 we list the total amount of useful data (i.e., data remaining after the removal of X-ray bursts and bad data of any kind) for each of these sources. In the case of Cir X-1, however, the quoted exposure time includes data affected by local absorption; see Section 3.3. Because of the large number of observations (over 2300 individual ObsIDs), most of the data analysis steps described below were automated.

The data were analyzed using HEASOFT, versions 6.9-6.12, as well as locally developed software. We used the Standard-2 data, extracting counts in $16 \mathrm{~s}$ time bins, combined from all three xenon layers, from all active Proportional Counter Units (PCUs) at any given time. The data were filtered following the recommendations of the RXTE Guest Observer Facility $(\mathrm{GOF}) ;{ }^{4}$ this included the removal of data around PCU voltage breakdown events (but only from the relevant PCU in each case). We also corrected for dead time using the standard procedure recommended by the RXTE GOF and subtracted background using the faint or bright background model as appropriate in each observation, based on the average count rate during the observation after exclusion of any type I X-ray bursts.

\subsection{Burst and Particle Flare Removal}

We removed all data obtained during type I X-ray bursts; to identify bursts in an automated fashion we used a method similar to the one used by Remillard et al. (2006). We note that although some bursts observed from Cyg X-2 and Cir X-1 do not show cooling along their tails-as is usually observed in type I X-ray bursts-the origin of these faint bursts is nevertheless very likely thermonuclear (Linares et al. 2011) and they were removed.

PCUs 0 and 1 lost their propane layers in 2000 and 2006, respectively, diminishing their ability to reject events due to particle background. Based on a comparison with data from PCU 2-which did not suffer from such particle flares and was nearly always on-these events were identified and subsequently removed from the data. We note that since PCU 2 data exist for all times during which PCU 0/1 data were removed, we did not exclude data from any given point in time entirely,

\footnotetext{
See http://heasarc.gsfc.nasa.gov/docs/xte/xhp_proc_analysis.html.
} 
but simply reduced the number of PCUs contributing to a particular time bin in these cases.

\subsection{Response Correction}

From the Standard-2 data we extracted count rates in several different energy bands and used those to calculate colors for CDs and HIDs. The response of the RXTE PCUs evolved over the duration of the mission due to several factors, and these changes in response must be corrected for before data across the lifetime of the mission can be combined into a single CD or HID for a given source. For this correction we used observations of the Crab Nebula, which was observed on average $\sim 3$ times per month throughout the mission. For each Crab observation, we extracted the average count rate in each of the energy bands of interest for each active PCU, using the Standard-2 data. We then fitted the mission-long light curve (where each data point represented the average count rate during a single observation) for each PCU in each energy band with a piecewise-linear function. We (somewhat arbitrarily) chose MJD 50800 (1997 December 18) as our reference epoch and normalized all data points to that date, first for each PCU individually, and then in each case the count rates from PCUs $0,1,3$, and 4 to that of PCU 2.

Recently it has become apparent that X-ray emission from the Crab Nebula shows significant intrinsic variability. Wilson-Hodge et al. (2011) show that from 2001 to 2010 the RXTE PCA count rate from the Crab (after correcting for changes in response) varied rather irregularly by several percent. The (response-corrected) PCU 2 variability is $\sim 5 \%$ in the $2-15 \mathrm{keV}$ band and $\sim 8 \%$ in the $15-50 \mathrm{keV}$ band. This can compromise our correction for the variation in the PCA response. Comparing our mission-long Crab light curves to those of Wilson-Hodge et al. (2011) we can estimate the magnitude of the effect on our derived rates and colors. The relative amplitude of the Crab count rate fluctuations gradually increases with energy, but otherwise the variability behaves in more or less the same way in the different energy bands. This energy dependence of the variability should have some effect on the colors; however, given the relatively small difference in the strength of the variability between adjacent energy bands, we expect that this variability will largely cancel out in the colors. We estimate that shifts in our colors due to the Crab variability are at most $\sim 1 \%-2 \%$ and in most cases significantly less; we therefore expect the influence of this on our results to be negligible (cf. discussion about uncertainty due to counting statistics in Section 2.4). The intensity is affected more strongly; we expect that shifts there can possibly be as high as $\sim 6 \%$ in the worst case. However, they are generally much smaller-probably less than $\sim 4 \%$ in almost all cases and typically in the $0 \%-3 \%$ range-and we expect the effect of this on our results to in general be negligible as well.

\subsection{Construction of CDs and HIDs}

For the creation of CDs (i.e., hard color versus soft color) and HIDs (hard color versus intensity) we used color definitions similar to those used in H10: we defined our soft color as the net counts in the $4.0-7.3 \mathrm{keV}$ band divided by those in the 2.4-4.0 keV band, and our hard color as net counts in the $9.8-18.2 \mathrm{keV}$ band divided by those in the $7.3-9.8 \mathrm{keV}$ band. The intensity we used for the HID was the net count rate

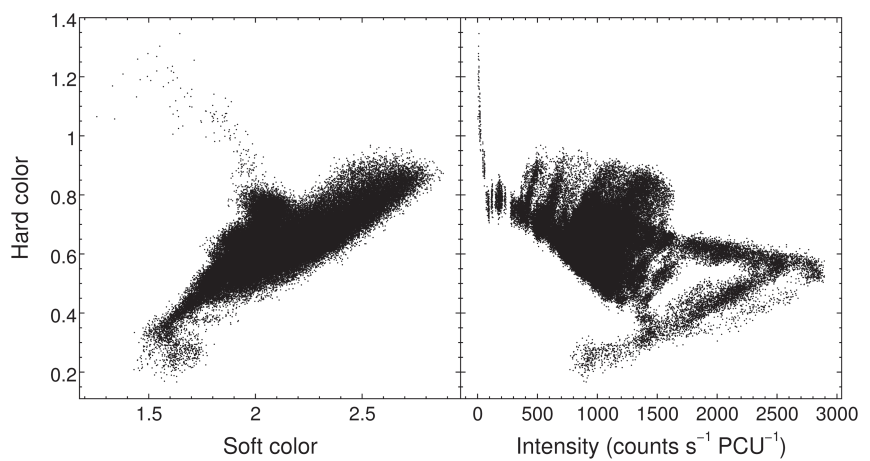

Figure 1. CD and HID representing the entire RXTE PCA data set of XTE J1701-462.

per PCU in the $2-60 \mathrm{keV}$ band. Before creating our CDs and HIDs we combined the counts from all active PCUs for each $16 \mathrm{~s}$ time bin (after performing the corrections and filtering described above) and then rebinned the data in a given observation in order to maintain a more uniform size in the error bars across different values in count rate. We set a minimum of 16,000 counts in the $2-60 \mathrm{keV}$ band (after background subtraction) for each rebinned data point. The rebinning was done in an adaptive/dynamic fashion-i.e., we in general did not use a single value for the time binning factor over an entire observation, but instead allowed the factor to vary (while imposing our counts limit) to adapt to a possibly varying count rate. In a few cases we applied a larger counts limit; these will be mentioned explicitly. In some instances entire observations did not contain enough counts to reach the 16,000 counts minimum; in those cases we created a single data point from the whole observation if the total number of counts was larger than 10,000 , but excluded it from further consideration if it had fewer counts. (Any exceptions to this will be explicitly mentioned.) We note that for most observations of the four bright sources studied here no rebinning was necessary, since each $16 \mathrm{~s}$ time bin usually contained more than the minimum 16,000 counts. With this minimum counts limit the uncertainty due to counting statistics is at most $\sim 2 \%$ in the soft color and at most $\sim 3 \%$ in the hard color (often much less); the uncertainty in the intensity is always less than $1 \%$.

\section{RESULTS}

\subsection{XTE J1701-462}

XTE J1701-462 was discovered with the RXTE All-Sky Monitor (ASM) on 2006 January 18 as the source was entering outburst (Remillard \& Lin 2006). The first RXTE PCA observation took place on 2006 January 19, and the source was subsequently observed with the PCA on average $\sim 1.5-2$ times per day for the remainder of the 19 month outburst, apart from a $~ 50$ day period in late 2006 and early 2007 during which the source could not be observed due to proximity to the Sun. The source returned to quiescence in early 2007 August and has since remained inactive apart from occasional low-level flaring (up to $\sim 10^{35} \mathrm{erg} \mathrm{s}^{-1}$ ), which has been observed by Swift and XMM-Newton (Fridriksson et al. 2010, 2011). As in H10, dates during the outburst will in this paper be referred to as days since the start of 2006 January 19 (MJD 53754.0). 

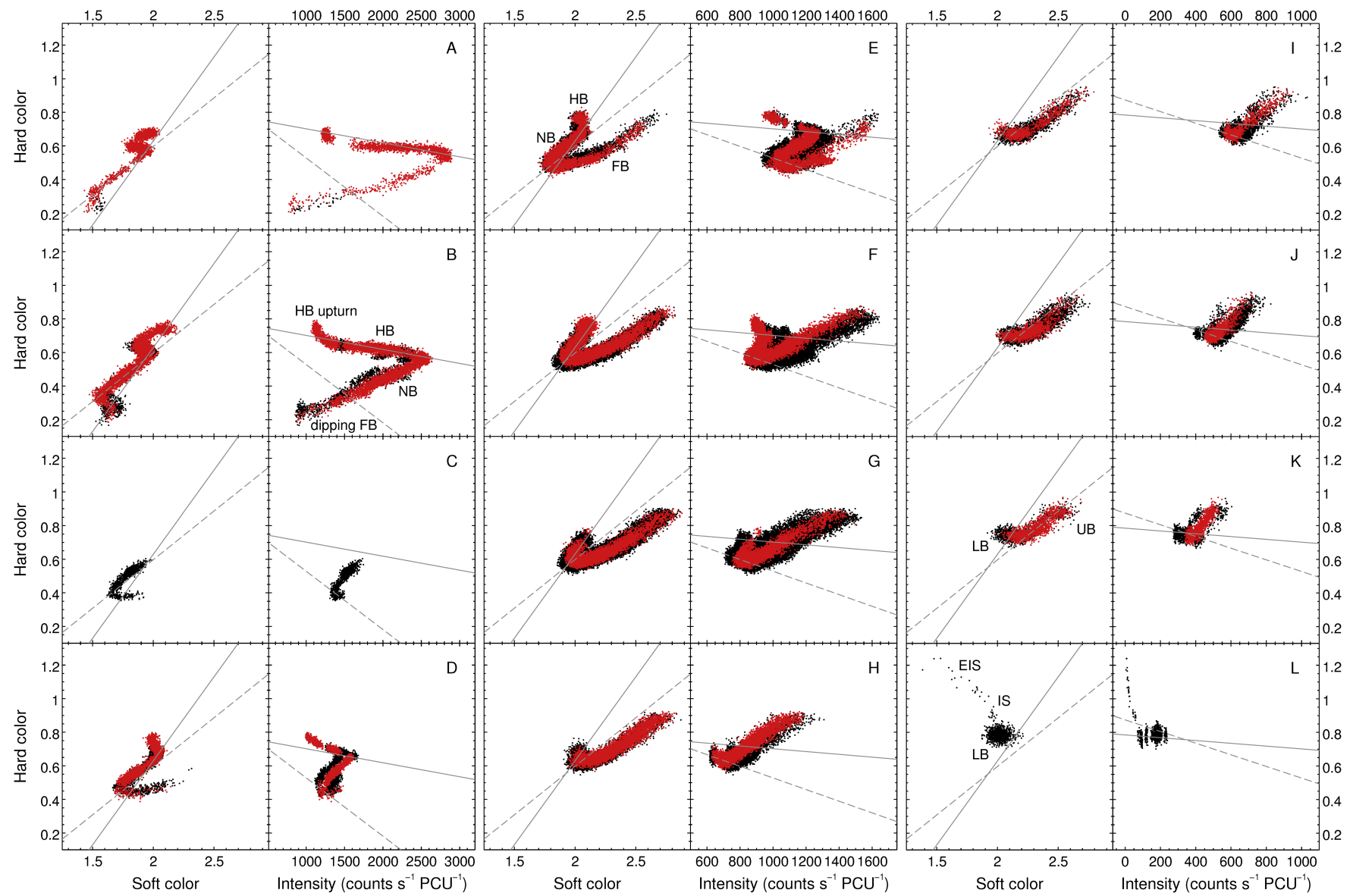

Figure 2. CDs and HIDs for XTE J1701-462, showing the secular evolution of the source. Each of the 12 panels (consisting of a CD on the left and an HID on the right) corresponds to a particular selection of data from the entire RXTE PCA data set for the source; red data points correspond to a particular subset (exhibiting minimal secular shifts) within a given selection (see Table 2). Particular source states/branches are indicated in a few of the panels (see text for definitions). The dashed and solid lines show the approximate paths followed by the NB/FB and HB/NB vertices, respectively, as the tracks evolve. Data are binned to a minimum of 16,000 counts per data point, except for the IS and EIS data in selection L, which are binned to a minimum of 64,000 counts. Note the change in the intensity scale between the different HID columns. We also note that essentially the same tracks (with only small modifications here) were presented in slightly different form in Figures 3 and 4 in H10.

The combined CD and HID for the entire 2006-2007 outburst of XTE J1701-462 are shown in Figure 1; the diagrams clearly illustrate that the source tracks exhibited strong secular motion during the outburst. As in $\mathrm{H} 10$ we divided the observations of the source into 12 subsets-hereafter referred to as selectionswhich we label A-L. In Figure 2 we show the CDs and HIDs for all 12 selections, which illustrate the secular evolution of the source during the outburst. We label the source states/branches of a few representative tracks: a Cyg-like Z track (selection B), a close-to Sco-like $\mathrm{Z}$ track (E), and two atoll-like tracks ( $\mathrm{K}$ and $\mathrm{L})$. For the Z-like tracks these are the horizontal branch (HB), the HB upturn, the normal branch (NB), the flaring branch (FB), and the dipping FB. For the atoll-like tracks these are the lower and upper banana branches (LB and UB), the island state (IS), and the extreme island state (EIS). The banana branch, IS, and EIS are also referred to as the soft, intermediate/transitional, and hard state, respectively. (We refer to H10 for examples of $\mathrm{CDs} / \mathrm{HIDs}$ of several Cyg/Sco-like $\mathrm{Z}$ sources and atoll sources and a comparison of those with the CD/HID tracks of XTE J1701-462.)

The 12 data selections mostly correspond to particular ranges in the low-energy $(2.0-2.9 \mathrm{keV})$ count rate, which H10 found to closely trace the secular changes during most of the outburst. This tracing of the secular evolution with the low-energy count rate breaks down when the source enters the $\mathrm{HB}$ or a dipping FB. For days 0-28 time-based selections (A and B) were therefore used, rather than ones based on the low-energy count rate. In addition, data from several $\mathrm{HB}$ excursions taking place after day 28 had to be moved to the same selection as neighboring NB data in the low-energy light curve. The time and count rate intervals we used are given in Table 2. Figure 2 in H10 shows a low-energy light curve of the entire outburst, indicating the count rate intervals used there (which are for the most part equivalent to ours) and the HB data moved between selections.

Within most of the selections some secular motion is still evident, mostly in the HID. We therefore in 10 of the 12 selections color red the data points from a subset of the observations used in each case, to show tracks with minimal secular shifts; the subintervals in time or count rate defining these subsets are given in Table 2. We also show, in both the CDs and HIDs, two straight lines that the NB/FB (lower) and HB/NB (upper) vertices of the tracks are seen to follow closely (as pointed out by Lin et al. 2009b). We note that selections A-L do not represent a strict monotonic progression in time (although overall the evolution in time was from A to L); the source moved back and forth between selections during the outburst, mostly within the range F-H (see 
Table 2

Time or Count Rate Intervals Used for Data Selections

\begin{tabular}{lcc}
\hline \hline Selection & $\left.\begin{array}{c}\text { Full Interval } \\
(\text { counts s }\end{array} \mathrm{PCU}^{-1}\right)^{\mathrm{a}}$ & $\begin{array}{c}\text { Subinterval } \\
(\text { counts s }\end{array}$ \\
\hline A $\left.\mathrm{PCU}^{-1}\right)^{\mathrm{a}}$
\end{tabular}

Note.

${ }^{\text {a }}$ Count rates are in the $2.0-2.9 \mathrm{keV}$ band. Time intervals refer to days since MJD 53754.0.

Figure 2 in H10). It is clear from the sequence in Figure 2 that going from panel A to $\mathrm{L}$ the tracks smoothly evolve in shape from Cyg-like $\mathrm{Z}$ to Sco-like $\mathrm{Z}$ to atoll tracks as the upper and lower vertices both move monotonically along the vertex lines to higher color values and the overall intensity of the tracks decreases.

After day 566 (as the outburst was ending) the count rate from the source reached a roughly constant level of $\sim 2$ counts $\mathrm{s}^{-1} \mathrm{PCU}^{-1}$ in the $2-60 \mathrm{keV}$ band $\left(\sim 0.2\right.$ counts $\mathrm{s}^{-1} \mathrm{PCU}^{-1}$ in the lowenergy band). This residual flux can be attributed to diffuse Galactic background emission (see Fridriksson et al. 2010). Figure 3 shows an alternative version of panel $\mathrm{L}$ in Figure 2 where we have binned the data more heavily and have subtracted this diffuse background emission based on data from observations made during the three weeks following the end of the outburst. In addition, we include in this version data from two observations made during days 564-566 (constituting the leftmost data point), which had a low-energy count rate of $\sim 0.36$ counts $\mathrm{s}^{-1} \mathrm{PCU}^{-1}$. We furthermore use a logarithmic scale for the horizontal axis in the HID so as to better illustrate the behavior of the source at the lowest count rates.

\subsection{Cyg $X-2$}

Cygnus X-2 (Cyg X-2) is one of the longest-known and most extensively studied X-ray binaries. Cyg X-2 was classified as a Z source by Hasinger \& van der Klis (1989) based on EXOSAT data and is the prototype of the Cyg-like subgroup of the persistent $\mathrm{Z}$ sources. However, as was clear already from pre- $R X T E$ data Cyg X-2 is unique among the six "classic" (persistent Galactic) Z sources in that it shows by far the strongest secular evolution (e.g., Kuulkers et al. 1996; Wijnands et al. 1997).

\subsubsection{Analysis}

In Figure 4 we show a CD/HID based on the entire $R X T E$ PCA data set for the source; strong variations in the shape and location of tracks are readily apparent. This combined CD/HID for Cyg X-2 has strong similarities to the one for XTE J1701-462 (Figure 1). However, the fragmented nature of the data set (obtained over a period of 15 years) forces us to

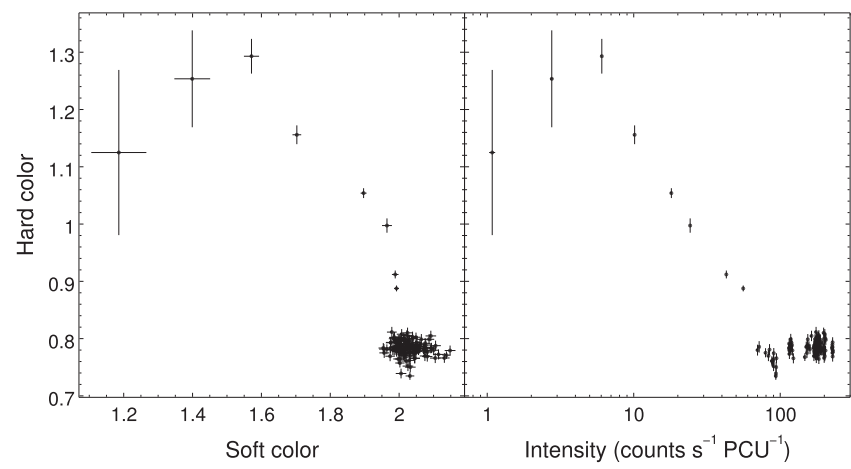

Figure 3. Alternative version of panel L in Figure 2 with the data binned into fewer groups and Galactic background emission subtracted. Data in the LB (i.e, intensity $\gtrsim 65$ counts $\mathrm{s}^{-1} \mathrm{PCU}^{-1}$ and hard color $\lesssim 0.85$ ) are binned to a minimum of 128,000 counts per data point; data in the IS and EIS are binned to approximately one data point per day. The data represented by the leftmost point were not included in Figure 2.

analyze the data in a manner different from the XTE J1701-462 analysis.

For this analysis we considered all 591 individual ObsIDs for pointed observations of Cyg X-2 made during the RXTE mission (13 of which contained no useful data). Often multiple observations were fairly densely clustered together in time. Going chronologically through the data set the source jumps erratically back and forth around the CD/HID, in any given observation usually tracing out only short partial track segments. As a first step we went through the entire data set in time order, at any point combining into a single CD/HID track as many consecutive ObsIDs as possible without introducing clearly noticable secular shifts. We refer to such clusters of data (containing all data from a certain time interval) as subsets. In a few cases significant secular motion took place during a single ObsID, requiring the observation to be split up between two different subsets. To identify secular motion we examined the tracks not only in the CD and HID, but also in a diagram of soft color versus intensity (which we refer to as an SID). The SID frequently yielded useful extra information/constraints. The result of this process was $\sim 300$ subsets, which span periods as long as several days, although the vast majority are less than a day in length.

Considering only individual subsets results in a few tracks that seem to a large extent complete, but most of the subsets consist of shorter segments (i.e., incomplete tracks). By an "incomplete" track we mean that had the source stayed long enough at a particular stage in its secular progression (and had

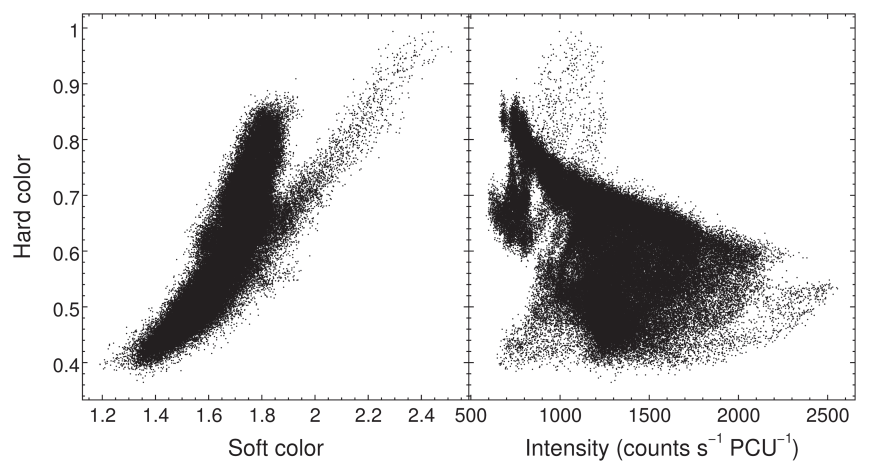

Figure 4. CD/HID representing the entire RXTE PCA data set of Cyg X-2. 

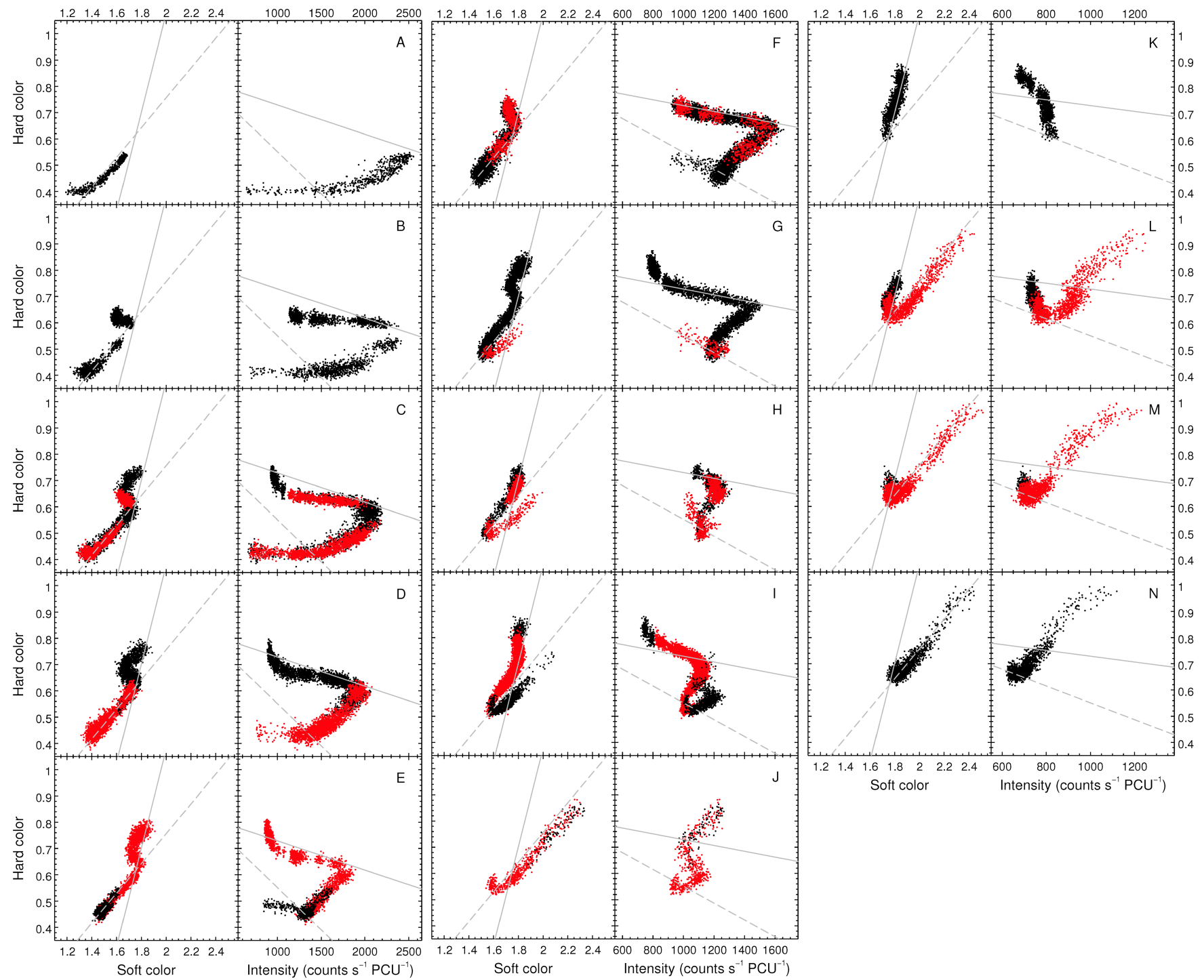

Figure 5. Sequence of CDs and HIDs for Cyg X-2 illustrating the secular evolution of the source. Table 3 lists the data used in each panel. In general, a panel combines data from observations widely separated in time (by months or years); exceptions to this are panels A, B, K, and N. In each of the other panels, a particular subset of the data - obtained over a period of at most a few days-is shown in red. The dashed and solid lines show the approximate paths followed by the lower and upper vertices, respectively. Note the change in the intensity scale between the different HID columns.

our observational coverage of the source been sufficiently comprehensive during that time) we expect that the source would have traced out a fuller (i.e., continuous and possibly more extended) track. It is important to note that this "completeness" is a function of location in the secular progression, as not all branches of the $\mathrm{Z}$ track are present at every stage of the secular evolution, and that there is always some uncertainty regarding what constitutes a "complete" track.

Fortunately, it was in general possible to combine subsets from various times throughout the RXTE mission to form more complete tracks than are available from individual subsets. Usually, the most complete individual-subset tracks served as a foundation on which these combined tracks were built. To guide us in this process we took advantage of overlapping track segments and made sure that they lined up in all three diagrams - the CD, HID, and SID — which together provided a fairly stringent criterion for the appropriateness of combining particular segments.
In Figure 5 we show a sequence of 14 tracks, chosen (from a larger set of tracks) to illustrate as clearly as possible the overall secular evolution exhibited by the source-both of the individual branches and the locations of the tracks-as they move through the CD/HID. As is the case for XTE J1701-462, the lower and upper vertices approximately follow straight line paths in both the CD and HID as the tracks evolve and shift in the diagrams. We show illustrative lines in Figure 5 and order the panels based on the vertex locations of the tracks, starting (panel A) at the highest intensities and lowest color values.

The number of individual subsets in the tracks in the Cyg X-2 sequence ranges from 1 to 10 . The subsets (i.e., time intervals) and ObsIDs used for each panel in Figure 5 are listed in Table 3. For the panels that consist of more than one data subset (i.e., all except $\mathrm{A}, \mathrm{B}, \mathrm{K}$, and $\mathrm{N}$ ), a single representative subset is plotted in red. The combined exposure time of the data shown in Figure 5 is $\sim 31 \%$ of the total exposure time of the Cyg X-2 data set (see Table 1). As far as we can tell, practically all the remaining data seem to belong 
Table 3

Time Intervals and Observations Used to Create Cyg X-2 Tracks

\begin{tabular}{|c|c|c|c|c|}
\hline Panel & Time Interval (MJD) & $\begin{array}{l}\text { Interval Length } \\
\text { days }(\mathrm{hr})^{\mathrm{a}}\end{array}$ & ObsIDs & $\begin{array}{l}\text { Exp. Time } \\
\text { ks }(h r)^{b}\end{array}$ \\
\hline A & $50316.592-50316.764$ & $0.17(4.1)$ & $10063-[09: 10]-01-00$ & $9.0(2.5)$ \\
\hline B & $51697.550-51698.705$ & $1.15(27.7)$ & 40019-04-05-[00:10] & $33.9(9.4)$ \\
\hline \multirow[t]{5}{*}{$\mathrm{C}$} & $50996.450-51000.593(\mathrm{R})$ & $4.14(99.4)$ & $30418-01-[(01: 05)-00,02-01]$ & $37.4(10.4)$ \\
\hline & $51561.344-51561.517$ & $0.17(4.1)$ & $40017-02-19-00$ & $10.1(2.8)$ \\
\hline & $53138.767-53138.855$ & $0.09(2.1)$ & $90030-01-16-00$ & $5.2(1.4)$ \\
\hline & $53788.496-53788.577$ & $0.08(2.0)$ & 91009-01-42-[00:01] & $2.7(0.7)$ \\
\hline & $54649.890-54650.626$ & $0.74(17.6)$ & 93443-01-01-[02,14:21] & $23.6(6.6)$ \\
\hline \multirow[t]{10}{*}{$\mathrm{D}$} & $51009.488-51009.725$ & $0.24(5.7)$ & $30046-01-01-00$ & $12.9(3.6)$ \\
\hline & $51349.631-51349.669$ & $0.04(0.9)$ & 40017-02-09-01 & $3.3(0.9)$ \\
\hline & $53079.687-53079.785$ & $0.10(2.3)$ & 90030-01-04-00 & $5.2(1.4)$ \\
\hline & $53222.440-53222.532$ & $0.09(2.2)$ & 90030-01-33-[00:01] & $4.8(1.3)$ \\
\hline & $53286.420-53286.512$ & $0.09(2.2)$ & $90030-01-46-00$ & $5.3(1.5)$ \\
\hline & $53291.340-53291.433$ & $0.09(2.2)$ & $90030-01-47-00$ & $5.4(1.5)$ \\
\hline & $54007.232-54007.331$ & $0.10(2.4)$ & 92039-01-15-00 & $5.7(1.6)$ \\
\hline & $54009.195-54010.341$ & $1.15(27.5)$ & $92039-01-[17,18]-00$ & $8.2(2.3)$ \\
\hline & $54452.324-54452.554$ & $0.23(5.5)$ & $90022-08-04-[00: 01]$ & $9.5(2.6)$ \\
\hline & $54648.066-54649.329(\mathrm{R})$ & $1.26(30.3)$ & 93443-01-01-[00,000,03:13] & $37.2(10.3)$ \\
\hline \multirow[t]{2}{*}{$\mathrm{E}$} & $50168.900-50169.731(\mathrm{R})$ & $0.83(20.0)$ & $10066-01-01-[00,000,001]$ & $37.3(10.4)$ \\
\hline & $51048.360-51048.599$ & $0.24(5.7)$ & $30046-01-07-00$ & $13.4(3.7)$ \\
\hline \multirow[t]{7}{*}{$\mathrm{F}$} & $51055.495-51055.782$ & $0.29(6.9)$ & $30046-01-08-00$ & $13.4(3.7)$ \\
\hline & $51266.719-51267.126$ & $0.41(9.8)$ & 40017-02-05-[00:01] & $15.9(4.4)$ \\
\hline & $51413.523-51413.746$ & $0.22(5.4)$ & $40017-02-12-00$ & $13.0(3.6)$ \\
\hline & $52429.372-52429.908$ & $0.54(12.9)$ & 70016-01-01-[02,04:06] & $12.4(3.4)$ \\
\hline & $53511.868-53512.546$ & $0.68(16.3)$ & $91010-01-01-[01,06: 09]$ & $29.9(8.3)$ \\
\hline & $53685.118-53685.193$ & $0.08(1.8)$ & $90030-01-82-00$ & $4.3(1.2)$ \\
\hline & $54395.861-54398.037(\mathrm{R})$ & $2.18(52.2)$ & $92038-01-[08-(00: 02), 09-(00: 02), 10-(00: 03)]$ & $12.3(3.4)$ \\
\hline \multirow[t]{10}{*}{ G } & $51081.447-51081.725$ & $0.28(6.7)$ & $30046-01-12-00$ & $14.3(4.0)$ \\
\hline & $51444.401-51445.215$ & $0.81(19.5)$ & 40019-04-[01-01G,01-02G,01-03,02-00,02-000] & $17.6(4.9)$ \\
\hline & $52533.929-52535.087$ & $1.16(27.8)$ & $70015-02-[01-01 \mathrm{G}, 02-00]$ & $19.1(5.3)$ \\
\hline & $53311.026-53311.105$ & $0.08(1.9)$ & $90030-01-51-[00: 01]$ & $2.6(0.7)$ \\
\hline & $53335.431-53335.724$ & $0.29(7.0)$ & $90022-08-01-00,90030-01-56-[00: 01]$ & $8.2(2.3)$ \\
\hline & $53375.032-53375.053$ & $0.02(0.5)$ & $90030-01-64-00$ & $1.8(0.5)$ \\
\hline & $53394.710-53394.812$ & $0.10(2.5)$ & $90030-01-68-00$ & $6.3(1.8)$ \\
\hline & $53488.223-53488.310$ & $0.09(2.1)$ & $91009-01-12-[00: 01]$ & $3.4(0.9)$ \\
\hline & $53493.152-53493.227(\mathrm{R})$ & $0.07(1.8)$ & $91009-01-13-[00: 01]$ & $2.8(0.8)$ \\
\hline & $53498.069-53498.142$ & $0.07(1.8)$ & $91009-01-14-00$ & $3.1(0.9)$ \\
\hline \multirow[t]{6}{*}{$\mathrm{H}$} & $51539.192-51539.562(\mathrm{R})$ & $0.37(8.9)$ & $40017-02-18-[00: 02]$ & $14.4(4.0)$ \\
\hline & $53119.069-53119.174$ & $0.10(2.5)$ & $90030-01-12-00$ & $6.8(1.9)$ \\
\hline & $53330.721-53330.805$ & $0.08(2.0)$ & $90030-01-55-[00: 01]$ & $2.9(0.8)$ \\
\hline & $53347.497-53347.527$ & $0.03(0.7)$ & $90022-08-02-00$ & $2.6(0.7)$ \\
\hline & $53744.187-53744.278$ & $0.09(2.2)$ & 90030-01-94-00 & $5.2(1.4)$ \\
\hline & $54388.862-54389.040$ & $0.18(4.3)$ & 92038-01-01-[00:02] & $3.8(1.1)$ \\
\hline \multirow[t]{5}{*}{ I } & $50629.832-50631.933(\mathrm{R})$ & $2.10(50.4)$ & 20053-04-01-[00:04,06,010,020,030 $]^{\mathrm{c}}$ & $77.5(21.5)$ \\
\hline & $51029.751-51030.032$ & $0.28(6.7)$ & $30046-01-04-[00: 01]$ & $13.8(3.8)$ \\
\hline & $53266.795-53266.821$ & $0.03(0.6)$ & $90030-01-42-00$ & $2.3(0.6)$ \\
\hline & $53758.990-53759.021$ & $0.03(0.7)$ & $90030-01-97-00$ & $2.7(0.7)$ \\
\hline & $54427.903-54428.970$ & $1.07(25.6)$ & 93082-02-04-[00:01] & $2.4(0.7)$ \\
\hline \multirow[t]{2}{*}{$\mathrm{J}$} & $53123.996-53129.015(\mathrm{R})$ & $5.02(120.5)$ & $90030-01-[13: 14]-00$ & $8.8(2.4)$ \\
\hline & $53320.893-53320.934$ & $0.04(1.0)$ & 90030-01-53-[00:01] & $1.4(0.4)$ \\
\hline $\mathrm{K}$ & $51528.887-51529.451$ & $0.56(13.5)$ & 40019-04-[03-01,04-(00:01),04-000] & $22.8(6.3)$ \\
\hline \multirow[t]{2}{*}{$\mathrm{L}$} & $50719.393-50719.759$ & $0.37(8.8)$ & 20057-01-01-[00,000] & $20.2(5.6)$ \\
\hline & $51536.473-51536.968(\mathrm{R})$ & $0.50(11.9)$ & $40021-01-02-[00: 04]^{\mathrm{d}}$ & $19.2(5.3)$ \\
\hline \multirow[t]{2}{*}{ M } & $51535.886-51536.436(\mathrm{R})$ & $0.55(13.2)$ & $40021-01-[01-01,02-00,02-000]^{\mathrm{d}}$ & $26.7(7.4)$ \\
\hline & $53202.754-53202.834$ & $0.08(1.9)$ & 90030-01-29-00 & $4.3(1.2)$ \\
\hline $\mathrm{N}$ & $51535.010-51535.571$ & $0.56(13.5)$ & $40021-01-01-[00,000,02]$ & $25.8(7.2)$ \\
\hline
\end{tabular}

Notes. Subsets colored in red in Figure 5 are denoted by $(\mathrm{R})$ in the Time Interval column. In the ObsIDs column a colon denotes a range.

${ }^{a}$ The interval length is shown in units of both days and hours.

b The total exposure time is shown in units of both ks and hours.

c As the source showed significant secular motion during observation 20053-04-01-04, only an early part of it was used.

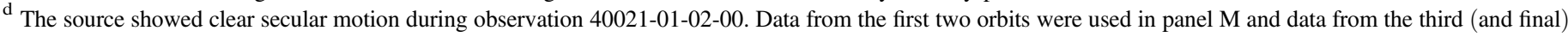
orbit was used in panel $\mathrm{L}$. 
to tracks similar to (and usually intermediate between) the ones shown in Figure 5 (i.e., none of the other subsets is inconsistent with belonging to such tracks).

Although the tracks in panels $\mathrm{K}$ and $\mathrm{L}$ do not line up well enough in the HID (contrary to the CD) to be combined into a single track, together they should give a reasonably good idea of the rough shape of a (near-)complete track at this point in the secular progression. It is clear that such a track is similar in shape to those of the Sco-like Z sources.

We note that small secular shifts do occur in some of these tracks; some of the individual segments used have a broad appearance (e.g., the red-colored segment in panel D), which is in some cases probably due to mild secular motion, and in some instances we matched up segments despite their not lining up perfectly in the HID or SID if the overall appearance of the track is only minimally affected by this. There was inevitably sometimes some ambiguity regarding whether certain segments used in a given track rather belonged to a track slightly shifted from the one in question. In particular, the HB upturn was usually observed in short isolated segments in the CD/HID, and it was often hard to judge exactly with which tracks those should be combined. However, the overall conclusions about the secular evolution of the source that we infer from the data are not sensitive to these ambiguities in the combining process. Finally, we note that many of the tracks in Figure 5 likely still suffer from some incompleteness, either because data segments that would serve to complete them are simply not available in the Cyg X-2 data set, or because we felt that there was too much ambiguity in whether candidate segments were appropriate. In particular, we conclude that the track in panel A is likely missing the HB, the tracks in panels B and $\mathrm{F}$ are presumably missing (most of) the $\mathrm{HB}$ upturn, the one in panel $\mathrm{H}$ is missing most of the $\mathrm{HB}$, the track in panel $\mathrm{J}$ is missing the $\mathrm{NB}$ and $\mathrm{HB}$, and the one in panel $\mathrm{K}$ is missing the FB.

\subsubsection{Comparison with XTE J1701-462}

Cyg X-2 exhibits secular evolution that is for the most part very similar to that of XTE J1701-462. As the overall intensity decreases the tracks smoothly evolve in shape from Cyg-like to Sco-like Z tracks. The tracks in Cyg X-2 panels A-E (Figure 5) are very similar to the Cyg-like Z tracks of XTE J1701-462 in panels A and B. Taken together, the tracks in Cyg X-2 panels K and $\mathrm{L}$ show that at this point in the secular progression of the source its tracks look similar to (and perhaps intermediate between) those in XTE J1701-462 panels E and F, the latter of which is very similar to the tracks of the persistent Sco-like Z sources, such as GX 17+2 (see, e.g., H10; Lin et al. 2012). Finally, the tracks in Cyg X-2 panels $\mathrm{M}$ and $\mathrm{N}$ look very similar to the tracks in XTE J1701-462 panels G and $\mathrm{H}$. As the Cyg X-2 tracks shift and evolve in shape, both the upper and lower vertices quite closely follow straight lines in the $C D$ and HID; as the overall intensity of the tracks decreases, the NB is squeezed between the converging vertex lines and gradually shortens, as observed in XTE J1701-462.

The main differences between Cyg X-2 and XTE J1701-462 lie in the fact that Cyg X-2 is persistently luminous and has never been seen to enter the atoll regime (although the track in panel $\mathrm{N}$ does not seem to have an NB, short NB segments seem to exist down to the very lowest intensities observed in the Cyg X-2 data set) and in the somewhat different FB behavior exhibited by Cyg X-2. In both sources the FB gradually evolves from being a purely dipping FB in the earliest (highestintensity, Cyg-like) panels to a "proper" flaring (Sco-like) FB in later panels. However, in Cyg X-2 the rotation of the FB is for the most part in the opposite direction to that seen in XTE J1701-462 in both the CD and HID. In Cyg X-2 the FB also develops a more complicated morphology in the HID, making a counterclockwise twist of between $180^{\circ}$ and $270^{\circ}$ around the lower vertex in panels $\mathrm{G}$ and $\mathrm{H}$ (such a twist was also observed in EXOSAT data by Kuulkers et al. 1996) and then assuming a jagged S-like shape in panels $\mathrm{I}$ and $\mathrm{J}$ that gradually straightens out going from panel I to N. We note that a similar, although less pronounced, S-shaped FB was observed in the HID of panel E in XTE J1701-462.

\section{3. $\operatorname{Cir} X-1$}

Circinus X-1 (Cir X-1) features some of the richest and most complex phenomenology seen among the known neutron star $\mathrm{X}$-ray binaries, and although it has been extensively studied for over four decades many of its properties remain poorly understood. The binary has an orbital period of $\sim 16.6$ days, identified from periodic flaring first observed in the X-ray band (Kaluzienski et al. 1976), and later in the radio, infrared, and optical (Whelan et al. 1977; Glass 1978; Moneti 1992). These flares are thought to be due to enhanced accretion near periastron passage in a highly eccentric orbit (e.g., Murdin et al. 1980; Tauris et al. 1999; Jonker \& Nelemans 2004). Heinz et al. (2013) identify the radio nebula surrounding the binary as a relatively young ( $\lesssim 5000$ yr old) supernova remnant, which makes Cir X-1 the youngest known X-ray binary and provides an explanation for the eccentricity of the orbit. This implies that the donor is likely an early-type star (see also Jonker et al. 2007). However, type I X-ray bursts detected from the source (Tennant et al. 1986a, 1986b; Linares et al. 2010) show that the neutron star is weakly magnetized, which is very unusual for a high-mass companion.

The X-ray emission from Cir X-1 is highly variable on a wide range of timescales. In Figure 6 we show an RXTE ASM light curve of the source. The source was in a historically high state during the first few years of the RXTE mission (Parkinson et al. 2003), with an average flux of $\sim 1.3$ Crab (and a maximum observed flux with the ASM of 3.5-4 Crab). The flux started gradually decreasing in mid-to-late 1999, and kept doing so until the source became undetectable with the ASM and showed no measurable activity over a two-year period in 2008-2010. In 2009 the source was observed with Chandra at a flux of only a few tenths of a milliCrab (Sell et al. 2010). Since 2010 May, however, the source has shown sporadic activity. When active the source usually exhibits complex variability over the course of an orbital period, featuring both absorption dips and flaring in the X-ray band; the effects of this on the ASM light curve can easily be seen in Figure 6. In the inset we show five orbital cycles from the bright phase of the source early in the RXTE mission. X-ray flaring is strongest during the first few days after it commences; it then gradually decreases in strength as the orbit progresses. The source usually exhibits strong X-ray dipping during the last $\sim 0.5-1$ days before the onset of flaring and then intermittent dips for up to two days afterwards (Shirey et al. 1998); we discuss this dipping in more detail below.

Oosterbroek et al. (1995) reported indications of both $\mathrm{Z}$ and atoll behavior in EXOSAT data of Cir X-1. Shirey et al. (1999a) analyzed data from 10 days of RXTE PCA observations in 


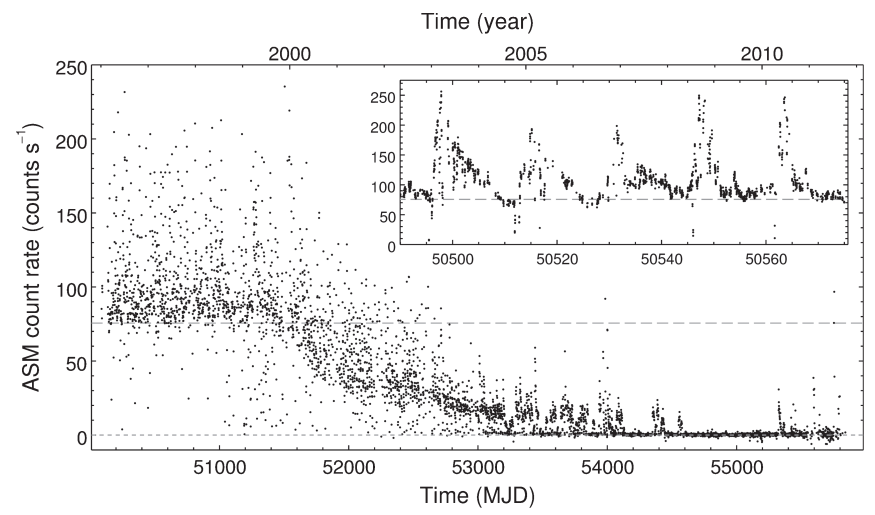

Figure 6. $R X T E$ ASM light curve of Cir X-1 covering the entire lifetime of the $R X T E$ mission. Data points in the main plot are one day averages. The inset zooms in on five orbital cycles in early 1997; each data point there corresponds to a single dwell of the ASM. The long-dashed lines in the main plot and inset show the typical ASM count rate level for the Crab Nebula.

1997. They detected all three Z branches_-although with some differences in shape compared to the classic $\mathrm{Z}$ sources-and found that the tracks moved around the CD/HID and evolved in shape. The identifications of the $\mathrm{Z}$ branches were supported by timing data.

\subsubsection{Analysis}

In our analysis we considered all 811 individual ObsIDs for pointed observations of Cir X-1 made during the RXTE mission (18 of which contained no useful data). A CD/HID using the entire data set (which spans $\sim 15$ years) is shown in the upper plot in Figure 7. The diagrams are heavily affected by both absorption and secular shifts and shape changes in the source tracks. As mentioned above, most of the dipping occurs close to the time of presumed periastron passage, which is often associated with flaring. Clarkson et al. (2004) fitted a quadratic ephemeris to the times of dips observed in RXTE ASM data from 1996 to 2003 and found that it provides a good predictor of the X-ray light curve. The strongest dipping often produces a characteristic track in the CD and HID; this track goes up to high color values and has two sharp bends in the CD. This can be seen in Figures 7 and 8; in the latter we show various diagrams for 7 days of observations in 1997 June, which we discuss further below. (The observations used in Figure 8 constitute the bulk of the data analyzed by Shirey et al. 1999a). The shape of these dipping tracks can be understood if the X-ray emission is composed of two components: a bright component that is subject to heavy absorption and a faint component that is unaffected by the absorption, the latter perhaps due to X-rays from the central source scattered into our line of sight by surrounding material (Shirey et al. 1999b). We note that the source also often exhibits shallower dips (also seen in Figure 8) which do not result in a (full) track of the sort described above.

Before proceeding with our analysis of the Cir X-1 data set we removed by eye (to the extent possible) data points affected by absorption dips. An example of this is shown in Figure 8, where removed data points are colored red. As can be seen in the figure, during these observations the source showed intense dipping shortly before the start of flaring (at day 611.5), producing the characteristic dipping track in the CD/HID. Some shallower dips were then observed during the first
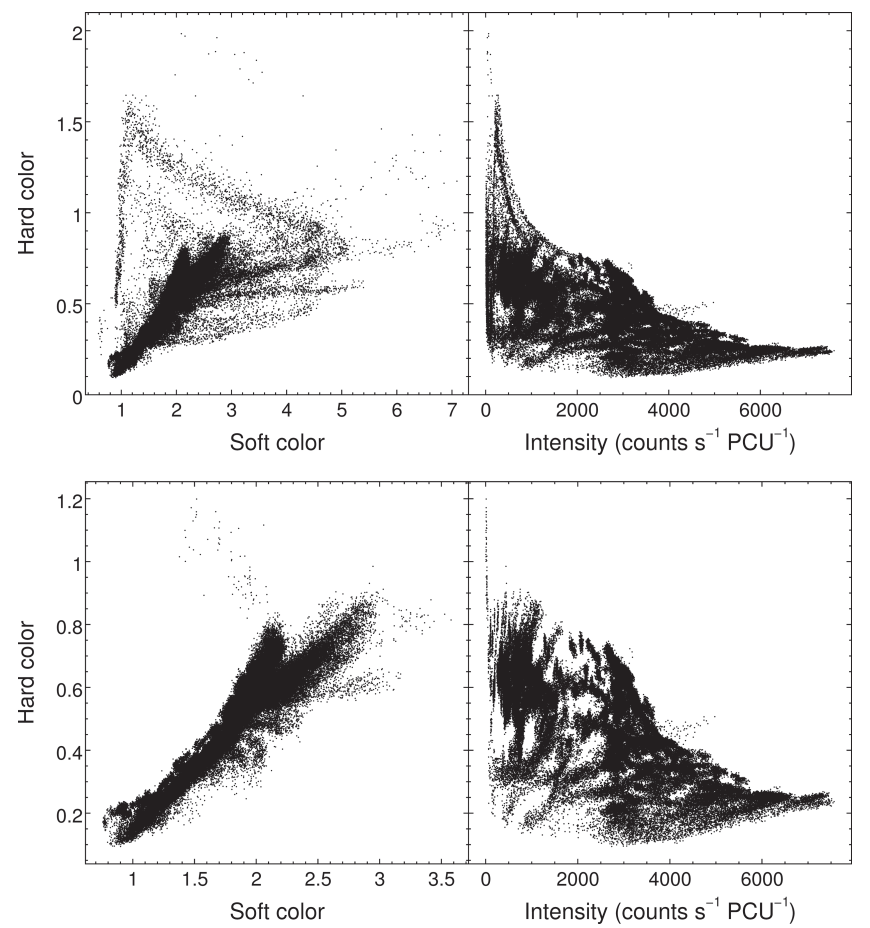

Figure 7. CD and HID representing the entire RXTE PCA data set of Cir X-1 before (upper plot) and after (lower plot) the removal of data affected by absorption. A small portion of the data at very high soft and hard color values falls outside the diagrams in the upper panel. Note the differences in scale in soft and hard color between the upper and lower panels.

$\sim 2$ days of flaring. We note that the periodic "flaring" during an orbital cycle is in general associated with motion along all three (Cyg-like) $\mathrm{Z}$ branches, rather than being exclusively associated with motion along the FB (which is mostly a dipping FB in these particular observations). As is apparent from the figure, it is almost impossible to identify absorption dips on the basis of the light curve alone during periods of flaring. However, tracking the behavior of the soft color (and, to a lesser extent, the hard color) as a function of time, as well as inspecting the $\mathrm{CD}$ and HID, greatly aids in identifying dipping. In addition, we took into account the dipping ephemeris of Clarkson et al. (2004) when performing the removal, since the vast majority of dipping events take place in the $\sim 1-1.5$ days immediately before or after phase 0 . However, we note that (shallow) dips are sometimes seen later in an orbital cycle; one example can be seen in Figure 8. In general, when unsure whether a given data segment was afflicted by dipping or not, we in general opted to rather err on the side of caution and remove the segment in question. The "cleaned" $\mathrm{CD} / \mathrm{HID}$ resulting from our manual removal of absorptionaffected data is shown in the lower plot in Figure 7. It was of course unavoidable that some instances of minor dipping remain and that a small amount of unaffected data be removed. However, we expect that the effects of this on the conclusions we draw from the data are negligible.

After removing data points affected by absorption dips, as described above, we organized the data into subsets, similar to our Cyg X-2 analysis described in the previous section. This resulted in $\sim 300$ subsets spanning periods as long as several days, although most are less than a day in length. From these, we created a sequence of $12 \mathrm{CD} / \mathrm{HID}$ (partial) tracks, shown in Figure 9. These tracks were chosen to illustrate as best possible 

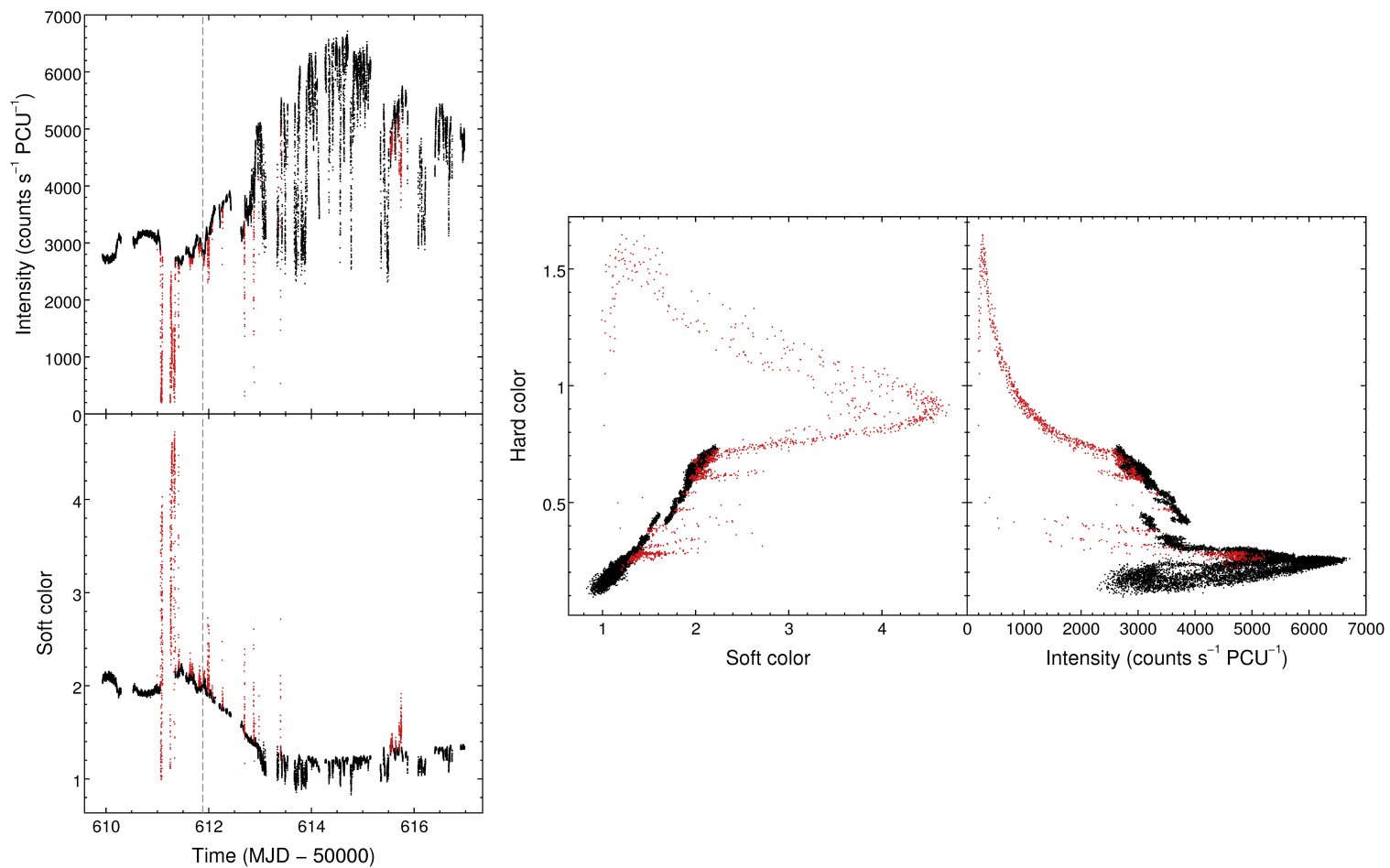

Figure 8. Diagrams demonstrating the removal of data affected by absorption from 7 days of observations of Cir X-1 in 1997 June; the left plot shows intensity and soft color as a function of time, and the right plot shows a CD/HID. We identified the red data points with absorption and removed them from the data set before proceeding with further analysis. In the left plot the dashed vertical line shows the time of zero phase according to the dipping ephemeris of Clarkson et al. (2004).

the overall secular behavior of the source. In all cases except two, the data in a given panel are from a single subset, whose time intervals range from $\sim 2 \mathrm{hr}$ to $\sim 7$ days. The exceptions are panels $\mathrm{A}$ and I, where 2-3 independent segments (shown in different colors) were combined. It was not viable to form a whole sequence of combined and more complete tracks, as we did for Cyg X-2, since the data for Cir X-1 show a much greater dynamic range and do not sample the HID densely enough for this. Overall, the upper and lower vertices of the Cir X-1 tracks move systematically up and to the right in the $\mathrm{CD}$, and up and to the left in the HID, as the tracks evolve in shape-similar to the behavior seen in XTE J1701-462 and Cyg X-2. The paths of the vertices can be approximated by the straight lines shown in Figure 9. We order the tracks based on the position of the lower and/or upper vertex in the $\mathrm{CD}$. (When both are present, the two vertices give consistent results.) In cases where some ambiguity remains due to very similar vertex locations (in particular, panels F and G), we use the shape of the track in the $\mathrm{CD}$ to decide the ordering, in particular the FB (i.e., we choose the ordering that produces a gradual evolution in the shape of the track in the CD). The combined exposure time of the data shown in Figure 9 is $\sim 10 \%$ of the total exposure time of the Cir X-1 data set. The data subsets (time intervals) and ObsIDs used in each panel are listed in Table 4.

We note that a few of the tracks in Figure 9 show some signs of secular motion in the HID (but much less in the CD); however, in most cases this has a negligible effect on the overall appearance of the track. The instance where this is most noticable is in the HID of panel E, where the upper parts of the track (NB and especially $\mathrm{HB}$ ) show shifting toward lower intensities relative to the FB. A similar, but smaller, shift affects the HID in panel F. We also note that the behavior of Cir X-1 is not as regular as that of XTE J1701-462 and Cyg X-2, and the vertices in the HID (and to a lesser extent in the CD) often deviate significantly from the lines shown. As will be discussed in Section 4, the relationship between a track's shape and its location in the HID is also not nearly as tight for Cir X-1 as for XTE J1701-462 and Cyg X-2, where the motion of both vertices in both the CD and HID, as well as the decrease in overall intensity, is monotonic along the entire sequence. The behavior of Cir X-1 is more regular in the $\mathrm{CD}$ than the HID, which is why we chose to use the CD as the basis for our ordering of the tracks. However, apart from some irregularities in panels $\mathrm{F}-\mathrm{I}$, the Cir X-1 sequence does show a gradual decrease in overall intensity.

Panel L shows what seems to be an atoll transition from the banana branch through the IS and to an EIS. These data were obtained in 2010 May/June as the intensity steadily decreased over a $\sim 7$ day period (part of a $\sim 50$ day minioutburst following two years of nondetection by the RXTE ASM). Observations preceding this 7 day period show what looks like a sequence of segments at successively lower count rates from tracks similar to the one in panel K. Figure 10 shows the same data as in panel L (in black) but with more binning. D'Aì et al. (2012) studied the spectral evolution of the source during the entire 2010 May-June minioutburst with spectral fitting and also concluded that the source transitioned from the atoll soft to hard state. In 2010 August the source showed a similar transition (again with an intensity decline over $\sim 7$ days), which we show with red data points in Figure 10. We note that the apparent decrease in hard color with decreasing intensity in the EIS in both cases may well be due to soft diffuse background emission (which could not be subtracted) affecting the data points at the lowest intensities. 


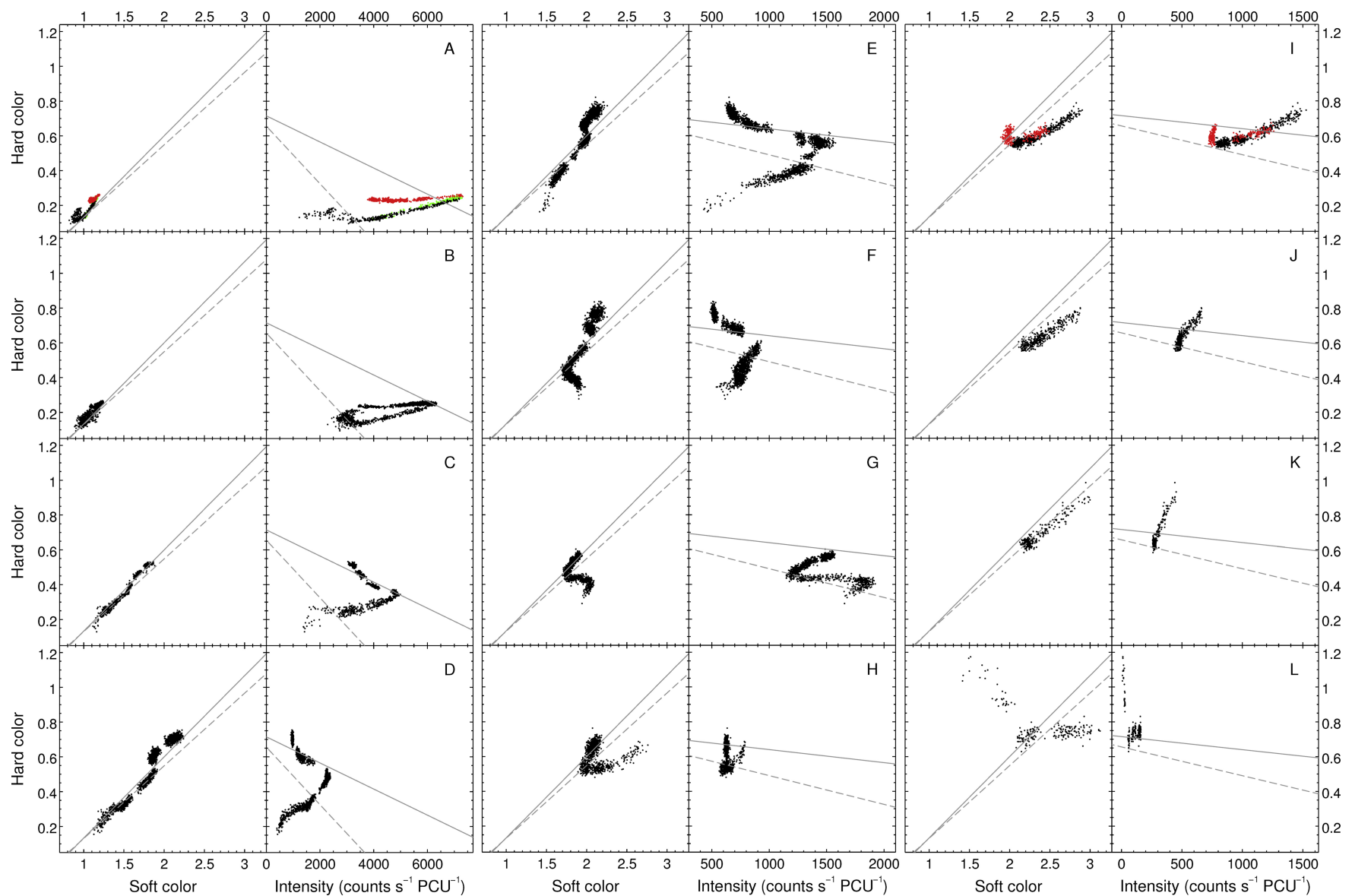

Figure 9. Sequence of CDs/HIDs for Cir X-1 illustrating the secular evolution of the source. The data points in each panel were obtained within a relatively short period of time, ranging from $\sim 4 \mathrm{hr}$ to $\sim 7$ days, except for panels A and I, which combine three and two segments, respectively, widely separated in time (with each segment shown in a particular color). The dashed and solid lines are lower and upper vertex lines similar to those shown for XTE J1701-462 and Cyg X-2. Data are binned to a minimum of 16,000 counts per data point, except for IS and EIS points in panel L, which are binned to a minimum of 32,000 counts. Note the change in the intensity scale between the different HID columns.

\subsubsection{Comparison with XTE J1701-462 and Cyg X-2}

Like XTE J1701-462, Cir X-1 has been observed in all NS-LMXB subclasses (Cyg-like Z, Sco-like Z, atoll). The overall secular evolution has many similarities to that observed for XTE J1701-462 and Cyg X-2, and many of the individual tracks have shapes similar to those seen for those two sources. Progressing along the Cir X-1 sequence, the NB grows shorter and rotates counterclockwise in the HID, while the HB rotates clockwise as it shortens, similar to what was seen for XTE J1701-462 and Cyg X-2. As in those two sources the FB shows the most complex behavior of the three branches in Cir X-1. In the CD of Cir X-1 the FB gradually rotates counterclockwise and evolves in shape in a similar fashion to XTE J1701-462. In the HID the behavior of the FB is more irregular in Cir X-1 than XTE J1701-462 and Cyg X-2, but overall it seems to rotate counterclockwise as it evolves from a dipping FB in the higher-intensity tracks to a Sco-like FB at lower intensities.

In panel G, the Cir X-1 FB in both the CD and HID has a shape similar to those of the persistent Cyg-like Z sources GX 340+0 and GX 5-1 (see, e.g., Jonker et al. 1998, 2002; H10). We note that in the CD of XTE J1701-462 the shape and orientation of the $\mathrm{FB}$ in panels $\mathrm{A}$ and $\mathrm{B}$ seems intermediate between Cir X-1 panels $\mathrm{E}$ and $\mathrm{F}$, whereas the
XTE J1701-462 FB in panels $\mathrm{C}$ (which has a very incomplete track) and $\mathrm{D}$ seems intermediate between Cir X-1 panels G and H. XTE J1701-462 may therefore have traced out a CD (and HID) track similar to those of GX 340+0 and GX 5-1 in between panels B and C; this portion of the secular progression of the source was missed due to a gap in RXTE coverage and rapid secular evolution. However, we note that in none of the four sources compared in this paper do we see a full track in both the CD and HID where the shapes of all three spectral branches closely match those of GX 5-1 and GX 340+0.

The atoll transitions of Cir X-1-especially the one in 2010 May/June-resemble the one of XTE J1701-462 (see Figures 3 and 10); one notable similarity is that the soft color decreases significantly throughout the transitions, in contrast to the increase observed in most atoll sources (e.g., Fridriksson 2011). However, there is also a striking difference between the atoll transitions of the two sources: the data from the banana branch preceding the ascent to the hard state extend to very high soft color for Cir X-1, occupying parameter space in the CD never explored before by the source in the RXTE archive except during absorption dips or (partly) during traversals to the tip of the (nondipping) FB. The data points in question are unlikely to be associated with the FB, given the small changes in intensity and hard color observed, and the long timescale 
Table 4

Time Intervals and Observations Used for Cir X-1 Tracks

\begin{tabular}{|c|c|c|c|c|}
\hline Panel & Time Interval (MJD) & $\begin{array}{l}\text { Interval Length } \\
\text { days }(\mathrm{hr})^{\mathrm{a}}\end{array}$ & ObsIDs & $\begin{array}{l}\text { Exp. Time } \\
\text { ks }(\mathrm{hr})^{\mathrm{b}}\end{array}$ \\
\hline \multirow[t]{3}{*}{ A } & $50365.216-50365.307$ & $0.09(2.2)$ & $10068-08-02-00$ & $5.5(1.5)$ \\
\hline & $50497.364-50497.437(\mathrm{G})$ & $0.07(1.8)$ & 20095-01-01-00 & $4.8(1.3)$ \\
\hline & $50711.692-50711.763(\mathrm{R})$ & $0.07(1.7)$ & 20095-01-18-00 & $5.9(1.6)$ \\
\hline B & $50613.800-50614.155$ & $0.36(8.5)$ & $20094-01-02-[04,040]^{\mathrm{c}}$ & $21.6(6.0)$ \\
\hline $\mathrm{D}$ & $52615.803-52618.276$ & $2.47(59.4)$ & 70020-01-[02-(01:02),03-01,03-04,04-(00:04)] & $37.9(10.5)$ \\
\hline $\mathrm{E}$ & $53013.822-53017.845$ & $4.02(96.6)$ & 70020-03-04-[00:20] & $34.6(9.6)$ \\
\hline $\mathrm{F}$ & $53163.821-53165.483$ & $1.66(39.9)$ & $80027-02-[02-02,02-06,03-(00: 02), 03-06]$ & $31.0(8.6)$ \\
\hline G & $51831.981-51832.459$ & $0.48(11.5)$ & 50136-01-04-[00:06] & $18.7(5.2)$ \\
\hline & $52951.992-52952.229$ & $0.24(5.7)$ & 80027-01-01-[02:03] & $9.4(2.6)$ \\
\hline $\mathrm{J}$ & $53003.507-53003.590$ & $0.08(2.0)$ & 70020-03-01-01 & $5.6(1.6)$ \\
\hline $\mathrm{K}$ & $53271.381-53271.787$ & $0.41(9.8)$ & $90025-01-02-[02,24,25,27]$ & $7.9(2.2)$ \\
\hline $\mathrm{L}$ & $55343.361-55350.716$ & $7.36(176.5)$ & $95422-01-[03-(03: 04), 04-(00: 13)]$ & $49.4(13.7)$ \\
\hline
\end{tabular}

Notes. Subsets colored in red/green in Figure 5 are denoted by $(\mathrm{R}) /(\mathrm{G})$ in the Time Interval column. In the ObsIDs column a colon denotes a range.

${ }^{a}$ The interval length is shown in units of both days and hours.

${ }^{\mathrm{b}}$ The total exposure time is shown in units of both ks and hours.

${ }^{c}$ The early parts of observations 20094-01-02-040 and 40059-01-01-00 were omitted due to secular motion.

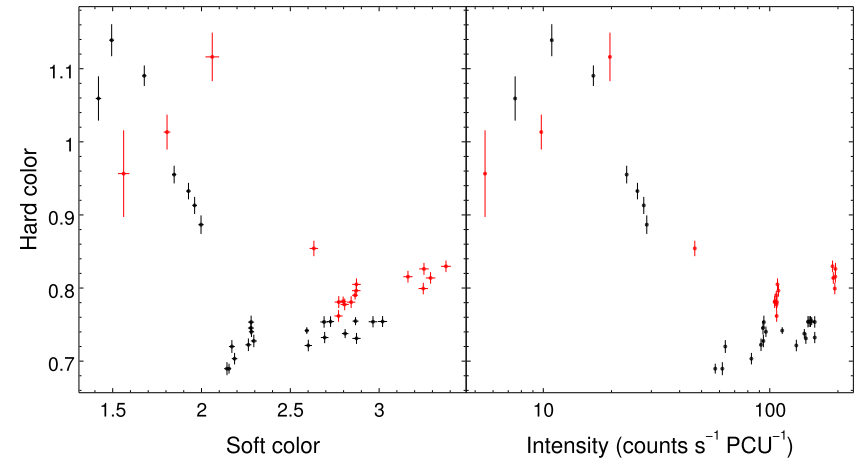

Figure 10. CD/HID showing two instances of Cir X-1 undergoing an atoll transition from the soft to hard state. Black data points are from a $\sim 7$ day period in 2010 May/June (also shown in panel L in Figure 9) and red data points are from a $\sim 7$ day period in 2010 August. The data were binned with a minimum of 128,000 counts per data point. In some cases entire observations did not have that many counts and were combined with other ones close in time.

involved (more than a day). This pre-atoll-transition behavior of Cir X-1 is in stark contrast to XTE J1701-462, where no such excursion to high soft color values was seen, and the region occupied by the source in the $\mathrm{CD}$ in selection $\mathrm{L}$ before moving to the IS was a logical extension of the movement of the source in the preceding selections. We also note that the observations in $2010 \mathrm{May} / \mathrm{June}$ were the first time in almost 15 years of RXTE observations that Cir X-1 was seen to transition to the atoll hard state.
The Cir X-1 track in panel D bears a strong resemblance in shape to the highest-intensity tracks (panels A and B) of XTE J1701-462 (in particular when the HID is plotted on the same scale) and occupies a similar color range (especially in hard color). But Cir X-1 also shows tracks that reach much higher intensities (panels $\mathrm{A}-\mathrm{C}$ ). Going from panel D to A in the Cir X-1 sequence, the overall color values of tracks decrease and the color range spanned drops sharply as the intensity swings become larger, resulting in very stretched and flat tracks in the HID. In these "extreme" Cyg-like Z tracks in panels A and B, Cir X-1 exhibits much lower color values than ever observed for any of the other three sources analyzed in this paper, and it is striking how small the color variations along the tracks are despite the large changes in intensity.

\section{4. $G X 13+1$}

GX $13+1$ is a bright X-ray binary whose classification as a $\mathrm{Z}$ or atoll source is ambiguous, although it has usually been labeled an atoll source. Hasinger \& van der Klis (1989) classified GX 13+1 as a bright atoll source based on CDs and power spectra from EXOSAT observations. In RXTE observations from 1996, Homan et al. (1998) discovered a $57-69 \mathrm{~Hz}$ QPO, which showed similar behavior to the horizontal-branch oscillation (HBO) seen in Z sources. Schnerr et al. (2003) performed a combined CD, HID, and powerspectral analysis of a large number of $R X T E$ observations made in 1998. They found that the source traced out, on a timescale of hours, a curved two-branched track in the CD, which 
showed strong secular motion on a timescale of $\sim 1$ week. The shape of the track was similar to the lower part (IS, LB, UB) of an atoll track or the NB/FB part of a $\mathrm{Z}$ track; the location of the vertex between the two branches was seen to approximately follow a straight line in the CD. They also found that the source showed peculiar CD/HID and rapid-variability behavior compared to most other $\mathrm{Z}$ or atoll sources, but overall they favored an atoll classification. Homan et al. (2004) analyzed two simultaneous RXTE/radio observations of GX $13+1$ performed in 1999. Based on the results of spectral fits, rapid-variability properties, behavior in the radio band, and the scarcity of type I X-ray bursts observed from the source since its discovery, they concluded that the properties of GX $13+1$ were more similar to $\mathrm{Z}$ sources than atolls.

\subsubsection{Analysis}

In our analysis we considered all 92 individual ObsIDs for pointed observations of GX $13+1$. These span a period of $\sim 14$ years. In Figure 11 we show a CD/HID based on all the GX $13+1$ data; strong secular motion is apparent. However, we note that GX $13+1$ overall shows the smallest range in secular evolution among the four sources studied here (e.g., as quantified by the range in soft or hard color over which the lower vertex is observed), and the other three sources show secular motion on shorter timescales than does GX 13+1. As for Cyg X-2 and Cir X-1 we organized the data into subsets; this resulted in $\sim 50$ such sets, which could in a few cases span intervals as long as several days with little or no visible secular motion. We constructed a sequence of six CD/HID tracks (shown in Figure 12), which illustrates the secular evolution of the source. Similar to our analysis of Cyg X-2, we combined subsets widely separated in time in three of these tracks to create more complete tracks than otherwise available and thereby give a fuller depiction of the overall secular behavior of the source. For these three tracks we also indicate in red a representative subset obtained in a short time interval. The number of individual subsets used for each track ranges from 1 to 4 ; these are listed in Table 5 along with the corresponding ObsIDs. Similar to our Cyg X-2 and Cir X-1 analysis, we order the tracks based on the location of the lower vertex in the CD, starting at the lowest soft and hard color. (See discussion of this vertex in the following paragraph.) The data used in the six tracks together constitute $\sim 42 \%$ of the total exposure time of the GX $13+1$ data set. We note that in a few of the subsets used we do see indications of small secular shifts-especially in panels B and F in Figure 12. These shifts are apparent mostly in the HID (and SID), rather than the CD. However, the overall conclusions we draw from these tracks and the sequence as a whole are not affected by this.

As can be seen from Figure 12 the tracks in the CD mostly have a two-branched form. The vertex between these branches follows rather closely a straight line (as observed by Schnerr et al. 2003), which we show in the figure. We classify GX $13+1$ as a $Z$ source based on its overall secular evolution in the CD/HID, the timescales on which it traces out its $\mathrm{CD} / \mathrm{HID}$ tracks, and its rapid-variability properties. (We discuss this further in Section 4.6.) We identify the vertex in the $\mathrm{CD}$ as the lower $\mathrm{Z}$ track vertex and the branches to the left/right of the vertex line in the $\mathrm{CD}$ as the normal/flaring branch. In addition, the tracks in panels $\mathrm{D}$ and $\mathrm{E}$ include subsets that we identify as the HB (plus upturn). In the CD these segments look very similar to excursions onto the $\mathrm{HB}$

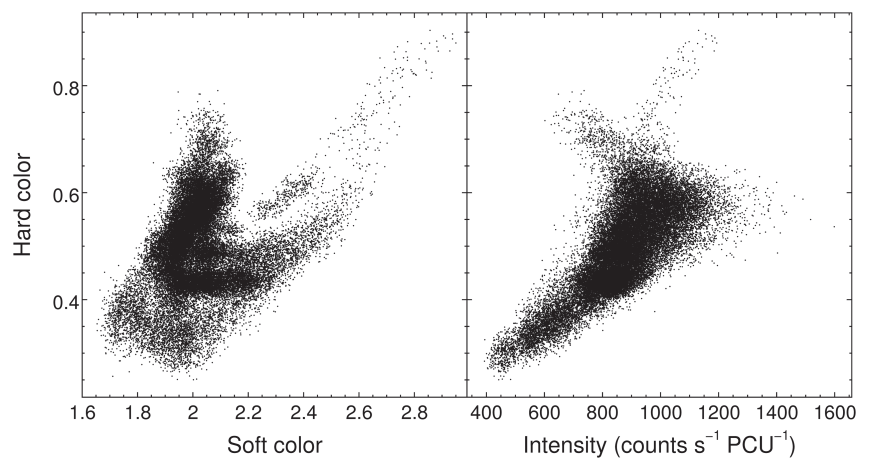

Figure 11. $\mathrm{CD}$ and HID representing the entire RXTE PCA data set of GX $13+1$.

and HB upturn in the Cyg-like Z tracks of XTE J1701-462, Cyg X-2, and Cir X-1. In the HID these segments also stand out and are located above and to the left of the rest of the track. We note that there is considerable ambiguity regarding where within panels $\mathrm{C}-\mathrm{E}$ to place these presumed $\mathrm{HB}$ segments. What we show in Figure 12 is therefore our best guess but only one of a few possible ways of incorporating them into the sequence. What does seem clear from inspection of the entire GX $13+1$ data set is that these segments cannot plausibly be combined with other track segments-or accommodated in our sequence-as NB segments similar to the other ones we see. (We discuss these tracks further in Section 4.)

The tracks in the HID look quite different from those of the other three sources. As we discuss below, different track branches can be identified in the HIDs; however, the fact that these branches are in general very broad and rather irregularly shaped makes them less useful for judging in what cases observations can be appropriately combined to form CD/HID tracks without significant secular shifts. The SID-although also displaying broad and somewhat irregular track branchesturned out to be more useful in this respect. To better illustrate the behavior of the source in the HID we show in Figure 13 a color-coded version of the track in panel $\mathrm{C}$. The locations of the $\mathrm{NB} / \mathrm{FB}$ vertex in the HID are in general less well defined than in the $\mathrm{CD}$ and they do not seem to follow a straight line.

\subsubsection{Secular Evolution}

GX 13+1 shows behavior that is in some ways quite different from that of the other three sources, especially in the HID. A more detailed description of the secular evolution of this source is therefore warranted. We note at the outset that, as Figure 11 indicates, the largest portion of the total exposure time for the source was spent on tracks near the middle of the sequence in Figure 12 (especially panel D), and it is therefore natural that the tracks in these panels would be the most complete ones. Conversely, the amount of data available at the lowest (panels A and B) and highest (panel F) vertex locations (all of which and is shown in Figure 12) is very small. These tracks therefore seem most likely to suffer from incompleteness.

We first focus on the evolution of the tracks in the CD. As the tracks move up the vertex line the FB gradually rotates counterclockwise and becomes longer and straighter (more Sco-like). Starting at the other end of the sequence, the NB gradually becomes longer as the tracks move down the vertex line from panel $\mathrm{F}$ to $\mathrm{D}$. The NB seems to shorten again going from panel $\mathrm{C}$ to $\mathrm{A}$, although we suspect that this may be due to 


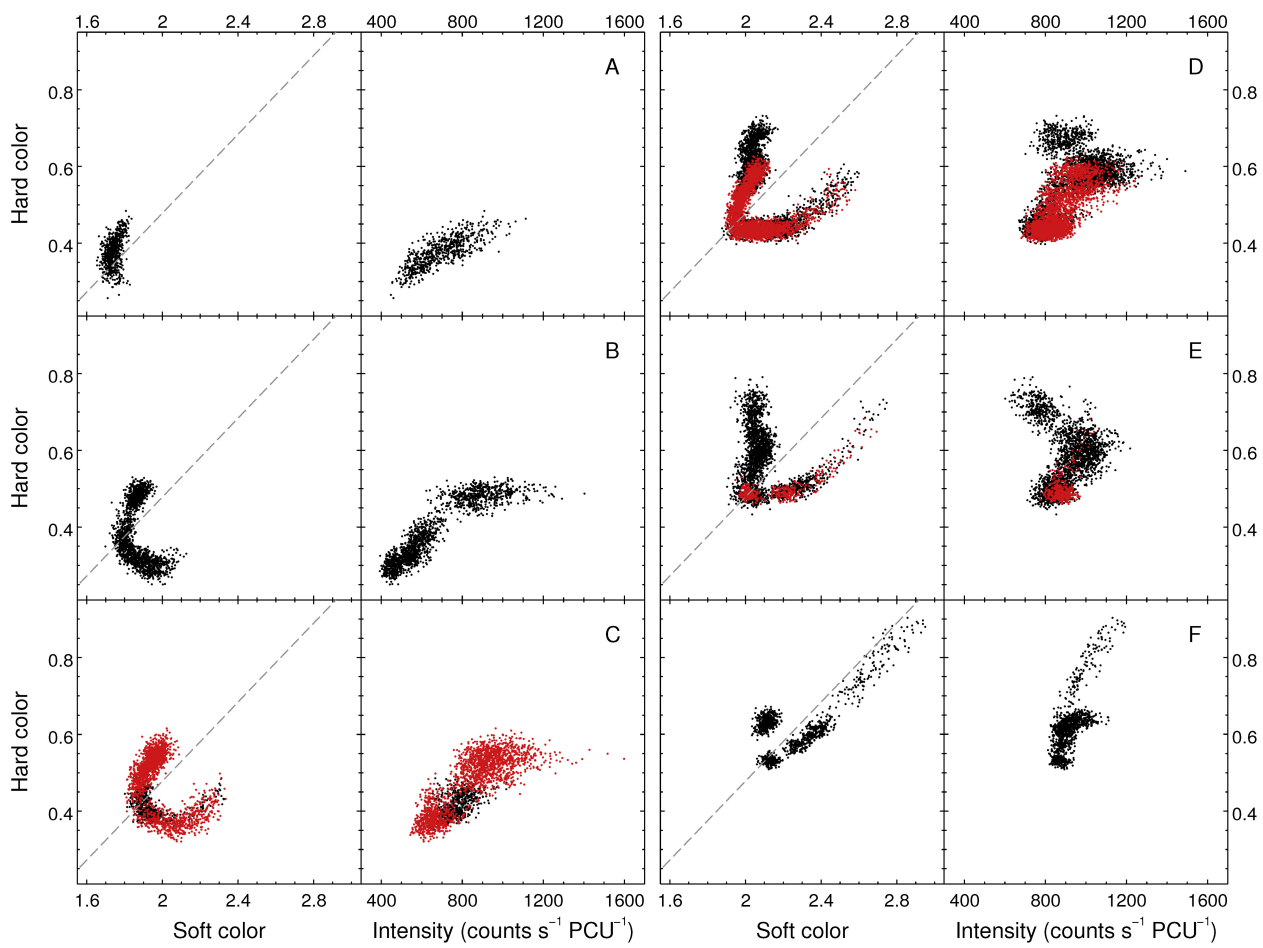

Figure 12. Sequence of CDs/HIDs for GX 13+1 illustrating the secular evolution of the source. Table 5 indicates the data used in each panel. In panels C, D, and E data from observations widely separated in time were combined; in each of these three panels a subset of the data—obtained over a period of at most $\sim 1.8$ days - is shown in red. The dashed line in the CDs shows the approximate path followed by the NB/FB vertex.

Table 5

Time Intervals and Observations Used for GX 13+1 Tracks

\begin{tabular}{|c|c|c|c|c|}
\hline Panel & Time Interval (MJD) & $\begin{array}{l}\text { Interval Length } \\
\text { days }(\mathrm{hr})^{\mathrm{a}}\end{array}$ & ObsIDs & $\begin{array}{l}\text { Exp. Time } \\
\text { ks }(\mathrm{hr})^{\mathrm{b}}\end{array}$ \\
\hline A & $51278.745-51278.971$ & $0.23(5.4)$ & 40023-03-02-03 & $10.3(2.9)$ \\
\hline $\mathrm{C}$ & $\begin{array}{l}50990.407-50990.711 \\
53767.006-53767.601(\mathrm{R})\end{array}$ & $\begin{array}{l}0.30(7.3) \\
0.60(14.3)\end{array}$ & $\begin{array}{l}30051-01-09-[00: 01] \\
91007-08-02-[00,000]\end{array}$ & $\begin{array}{r}9.8(2.7) \\
29.5(8.2)\end{array}$ \\
\hline $\mathrm{E}$ & $\begin{array}{l}51007.409-51007.560 \\
53409.846-53409.949(\mathrm{R}) \\
54740.627-54743.339 \\
55409.062-55413.805\end{array}$ & $\begin{array}{l}0.15(3.6) \\
0.10(2.5) \\
2.71(65.1) \\
4.74(113.8)\end{array}$ & $\begin{array}{l}30051-01-12-01 \\
90173-01-01-00 \\
93046-08-[02-00,03-(00: 01)] \\
95338-01-[01-(00: 07), 03-00,03-05]\end{array}$ & $\begin{aligned} 8.0 & (2.2) \\
6.1 & (1.7) \\
7.8 & (2.2) \\
24.3 & (6.8)\end{aligned}$ \\
\hline
\end{tabular}

Notes. Subsets colored in red in Figure 12 are denoted by $(\mathrm{R})$ in the Time Interval column. In the ObsIDs column a colon denotes a range.

${ }^{a}$ The interval length is shown in units of both days and hours.

${ }^{b}$ The total exposure time is shown in units of both ks and hours.

incompleteness in panels A, B, and possibly C. These three tracks may also be missing $\mathrm{HB}$ and $\mathrm{HB}$ upturn portions.

The evolution in the HID is less obvious. At the lower vertex locations the NB is strongly tilted to the right and for a given hard-color value spans a large intensity range, especially in its upper part. The NB gradually becomes more compact going to higher vertex locations (although it is always broad compared to the NBs observed in the other three sources). The behavior of the FB in the HID is even harder to discern than that of the NB. The morphology of the FB in panel C can more easily be seen in the color-coded version in Figure 13. The upwardpointing part of the FB is essentially vertical (but very short) in panel $\mathrm{C}$, and is at the lowest intensities. Going counterclockwise along this track in the $\mathrm{CD}$, the highest intensities are near the top of the NB and then the intensity becomes gradually lower moving along the track until the FB reaches its lowest 


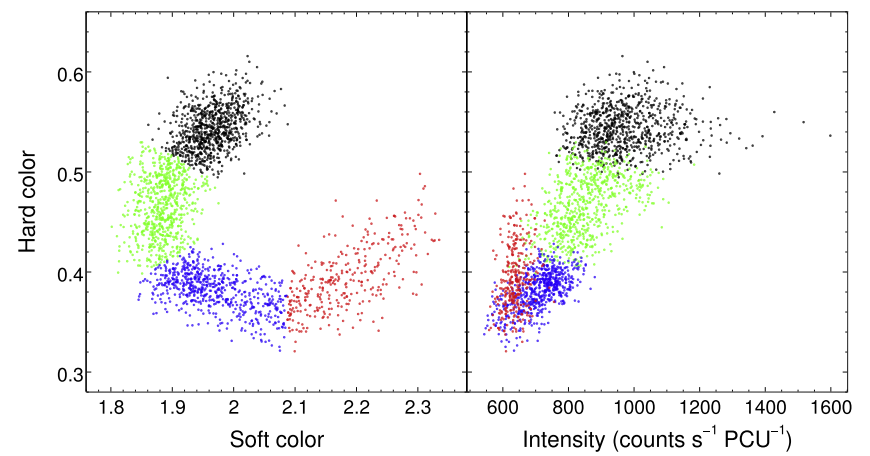

Figure 13. Color-coded version of the track in panel $\mathrm{C}$ in Figure 12, illustrating the portions of the HID track corresponding to several segments along the track in the CD.

point (in hard color), after which the intensity becomes approximately constant. (The same behavior is seen in panel B.) In panels $D$ and $E$, the flat part of the FB in the $\mathrm{CD}$ forms a broad patch at the bottom of the track in the HID. The rising part of the FB extends from the left side of this patch, tilted slightly to the right from vertical, and overlaps with the broad NB (see, e.g., the red-colored data in panel E).

\subsubsection{Comparison with XTE J1701-462, Cyg X-2, and Cir X-1}

The tracks in the CD of GX 13+1 show many similarities to those of XTE J1701-462, Cyg X-2, and Cir X-1. The evolution of the FB in the CD is similar to that seen for XTE J1701-462 in selections $\mathrm{A}-\mathrm{H}-$ with the $\mathrm{FB}$ rotating counterclockwise and growing longer (and more Sco-like) - and likewise similar to that of Cir X-1. There are strong similarities between the shape of the FB of GX $13+1$ in panels D-F and, e.g., the FB of XTE J1701-462 in selections E-H, Cyg X-2 in panel J, and Cir X-1 in panels $\mathrm{H}$ and I. As in the other three sources, a lower vertex in the $\mathrm{CD}$ of GX $13+1$ moves along a straight line with positive slope. The NB is also similar to the ones of the other three sources (being mostly straight and oriented up and to the right), and the possible $\mathrm{HB}$ and $\mathrm{HB}$ upturn segments in GX $13+1$ have a similar appearance to those of the other sources as well.

Although less obvious at first sight, there are also similarities in the HID between GX $13+1$ and the other three sources. The NB is oriented up and to the right, becoming gradually shorter and closer to vertical as the tracks move up in hard color-i.e., the intensity swings along the NB become smaller-and at the same time the maximum intensity observed on the NB gradually decreases (although for this to extend to panels $\mathrm{A}$ and $\mathrm{B}$ we need to assume they have incomplete NBs). This is also the case for the other three sources. What we interpret as traversals onto the $\mathrm{HB}$ and $\mathrm{HB}$ upturn in GX 13+1 are manifested as movement toward lower intensities and higher hard color; this is in general the case for $\mathrm{Z}$ sources, including the other three discussed in this paper. In the earlier panels in the GX $13+1$ sequence the initial part of the FB is toward lower intensities (i.e., dipping FB behavior), as is seen in the other three sources. The GX 13+1 FB rotates slightly clockwise in the HID (similar to Cyg X-2) as the source moves up the vertex line in the $\mathrm{CD}$, evolving into an FB that shows an intensity increase rather than decrease. A notable difference between GX $13+1$ and the other three sources is the movement of the lower vertex in the HID. The location of the lower vertex in the HID of GX $13+1$ is in general rather poorly defined, and does not seem to follow a straight line. However, it seems clear that the lower vertices in panels $\mathrm{A}$ and $\mathrm{B}$ in Figure 12 are at a significantly lower intensity $\left(\sim 550-600\right.$ counts $\left.\mathrm{s}^{-1} \mathrm{PCU}^{-1}\right)$ than the ones in panels $\mathrm{C}-\mathrm{F}\left(\sim 800-900\right.$ counts $\left.\mathrm{s}^{-1} \mathrm{PCU}^{-1}\right)$, in contrast to the other three sources, where in general the intensity at the lower vertex decreases as it moves up the vertex line in the CD.

As is clear from the above GX $13+1$ shows similarities to both Cyg-like and Sco-like Z sources. Overall, the earlier panels in the track sequence are more Cyg-like and the later ones more Sco-like, although the distinction between the two is less clear than for the other three sources.

\section{DISCUSSION}

\subsection{Secular Evolution}

The main goal of this paper is to study secular evolution in the CDs and HIDs of NS-LMXBs, using three sources historically known to show substantial changes in the shape and location of their CD/HID tracks: Cyg X-2, Cir X-1, and GX $13+1$. In particular, we want to determine to what extent the secular evolution of these three sources is similar to that seen in XTE J1701-462, the first source found to evolve through all NS-LMXB subclasses.

In Figure 14 we provide an overview of the secular evolution of the CD/HID tracks of XTE J1701-462, Cyg X-2, Cir X-1, and GX $13+1$. In each of the four panels we show all tracks from the sequences in Figures 2, 5, 9, and 12 in a single CD/HID. (For XTE J1701-462 we show only the red-colored subsets from Figure 2, where applicable.) Strong secular evolution is found in all four sources, consistent with reports in the literature. While for XTE J1701-462 all data come from the dense monitoring of a single 19 month outburst, the data for the other three sources were collected sporadically over a time span of $14-15$ years. As a result, the secular evolution in these sources could not be followed in "real time," as was possible for XTE J1701-462, but had to be reconstructed from multiple isolated (partial) tracks. To some extent this limited our ability to compare the secular evolution between sources, in particular for GX 13+1. Despite these issues, it is obvious from Figure 14 that the secular evolution of the four sources has many common characteristics.

In the following we summarize our key findings, focusing on the similarities between the systems.

1. As part of their secular evolution, we see clear and continuous transitions between different NS-LMXB subclasses in all four sources. While some of the behavior we report has been described in previous works, our work for the first time unambigously links strong secular evolution to transitions between various NS-LMXB subclasses in Cyg X-2, Cir X-1, and GX 13+1. Of the four sources XTE J1701-462 and Cir X-1 have shown the largest range in behavior; they have displayed Cyg-like and Sco-like $\mathrm{Z}$ tracks, atoll soft and hard states, and have both at some point entered quiescence or near-quiescence $\left(\sim 10^{35} \mathrm{erg} \mathrm{s}^{-1}\right.$ in the case of Cir X-1; Sell et al. 2010). Cyg X-2 and GX 13+1 have only shown Cyg-like and Sco-like Z tracks, with the secular changes in GX $13+1$ being the most moderate of the four sources.

2. Cyg-like $Z$ source behavior (with large variations in intensity along the NB and $\mathrm{HB}$, and a "dipping" FB that 

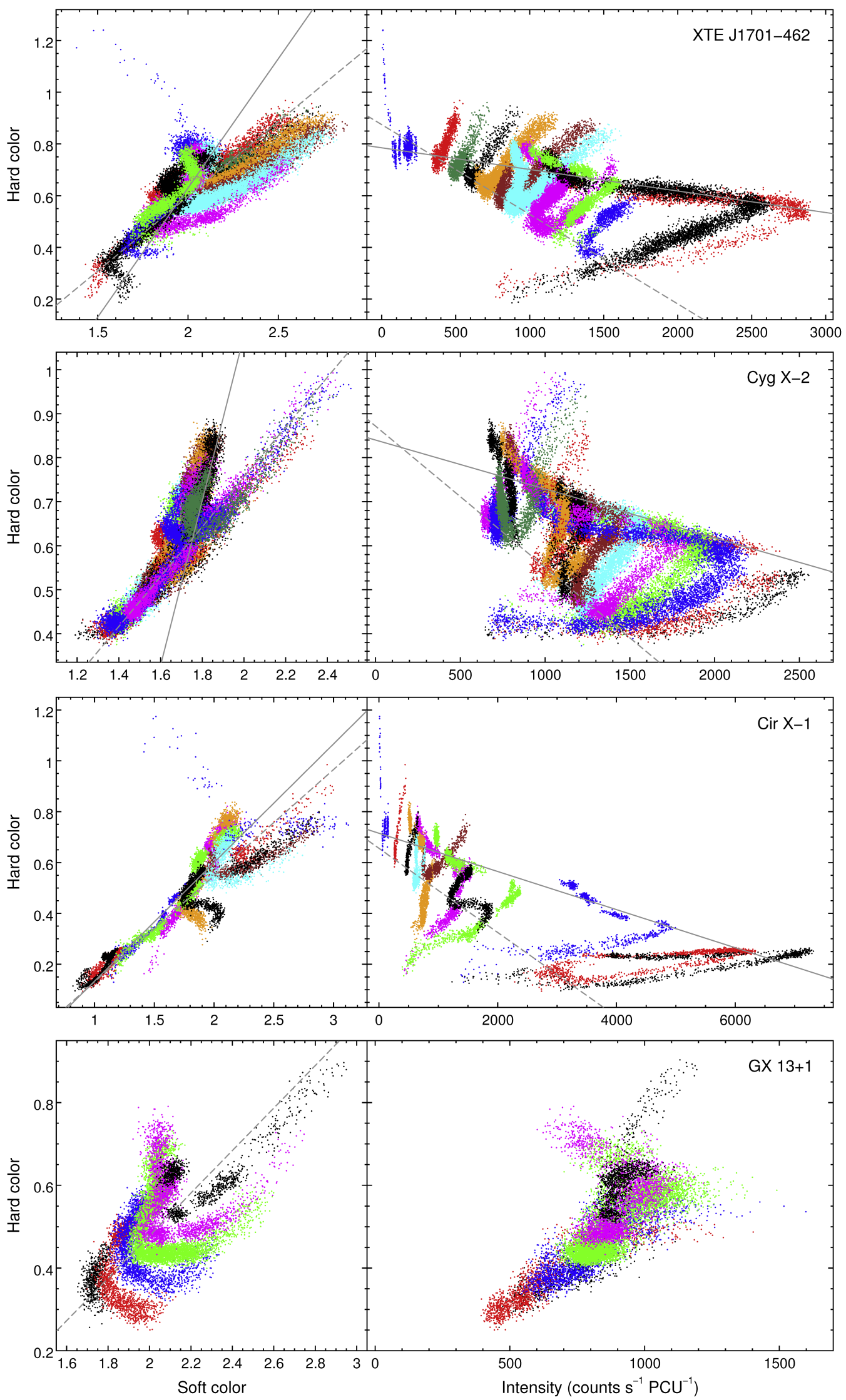

Figure 14. CDs/HIDs illustrating the overall secular evolution of XTE J1701-462, Cyg X-2, Cir X-1, and GX 13+1. In the panels we show (from top to bottom) all the tracks from Figures 2, 5, 9, and 12, respectively. Different colors are used to distinguish between the individual tracks in each panel. 
is directed toward lower intensities) is observed at the highest overall intensities, Sco-like Z source behavior (with small intensity variations along the NB and, if present, the $\mathrm{HB}$, and large increases in intensity along the FB) at lower intensities, and (in XTE J1701-462 and Cir X-1) atoll behavior at the lowest overall intensities.

3. Although possible incompleteness in some of the CD/HID tracks clouds the picture somewhat, the order in which the $\mathrm{Z}$ source branches evolve and disappear in Cyg X-2 and Cir X-1 seems to be consistent with what is seen in XTE J1701-462. As the overall intensity decreases, the HB shortens and disappears first, followed by the NB, and finally the FB. The situation is less clear for GX 13+1; the earlier tracks in Figure 12 would need to be missing an $\mathrm{HB}$ (and possibly part of the NB) for the behavior of GX $13+1$ to be consistent with XTE J1701-462 as well.

4. As the shapes of the $Z$ tracks and branches gradually change, the vertices of the tracks shift along well-defined lines in the $\mathrm{CD}$, moving toward higher color values as the overall intensity decreases. (We note, however, that the overall intensity evolution is less clear-cut for GX 13+1, and that Cir X-1 shows some deviations from the general intensity trend.) For XTE J1701-462 and Cyg X-2 this also applies to the HID, and to a large extent for Cir X-1 as well.

5. As the overall intensity of the $\mathrm{Z}$ tracks increases and they become spectrally softer, their dynamic range (i.e., ratio of maximum to minimum intensity) increases as well. Whether this trend also extends to the tracks at the lowest color values in GX $13+1$ is unclear due to possible incompleteness issues.

Despite the fact that differences exist in their detailed behavior, based on the above findings we conclude that the secular evolution in the four NS-LMXBs that we studied largely follows similar patterns. This strongly suggests that the sequences of NS-LMXB subclasses reported in this work are representative of the class of NS-LMXB as a whole.

\subsection{Evolution of the Flaring Branch}

Of the three main $\mathrm{Z}$ source branches, the FB undergoes the most dramatic changes in its shape and orientation. In all four sources it rotates in both the CD and HID and evolves from a branch that shows a strong decrease in intensity with respect to the $\mathrm{NB} / \mathrm{FB}$ vertex (in the Cyg-like $\mathrm{Z}$ tracks), to one that shows a strong increase in intensity with respect to that vertex (in the Sco-like $\mathrm{Z}$ tracks), although the amplitude of these intensity changes is significantly smaller in GX $13+1$ than in the other three sources. From the dense "coverage" of the secular evolution of XTE J1701-462 and Cyg X-2 it is clear that this FB evolution from dipping to flaring is a very gradual process, with the morphology changing smoothly throughout the track sequences. This suggests that the dipping Cyg-like FB and the Sco-like FB are related phenomena, and it seems unlikely that the overall observed FB behavior can be explained by two presumably unconnected mechanisms: absorption by the outer disk, which was proposed as an explanation for the Cyg X-2 dipping behavior by BałucińskaChurch et al. (2011), and nuclear burning on the neutron star (combined with increases in the mass accretion rate in the Sco-like Z sources), proposed as an explanation for the flaring
FB by Church et al. (2012). (See also Bałucińska-Church et al. 2012.)

\subsection{Other Z Source Transients}

We note that two additional $\mathrm{Z}$ source transients discovered after we began work on this paper, IGR J17480-2446 (also known as Terzan 5 X-2) and MAXI J0556-332, have shown similar behavior to the sources studied here. $R X T E$ observations of IGR J17480-2446 revealed clear Cyg-like Z source behavior at the peak of its 2.5 month outburst and atoll behavior at lower intensities; due to the rapid source evolution and sparsity of the data Sco-like $\mathrm{Z}$ behavior could not be identified unambiguously (Altamirano et al. 2010, 2012; Chakraborty et al. 2011; D. Altamirano et al. 2015, in preparation).

MAXI J0556-332 showed both Cyg-like and Sco-like Z behavior during its 16 month outburst (Homan et al. 2011, 2014; Sugizaki et al. 2013; J. Homan et al. 2015, in preparation). Figure 2 in Homan et al. (2014) shows a combined HID using all the RXTE data for the source (panel a) as well as four timeselected HID tracks illustrating the secular evolution of the source (panels b-e). The total HID looks similar to that of Cyg X-2 (Figure 4). The higher-intensity MAXI J0556-332 tracks (panels $\mathrm{b}$ and c) are Cyg-like $\mathrm{Z}$ tracks; the track in panel b shows an extended dipping FB and resembles the highest-intensity tracks of XTE J1701-462, Cyg X-2, and Cir X-1. The lower-intensity tracks of MAXI J0556-332 (panels d and e) are Sco-like Z tracks, resembling the tracks in panels $\mathrm{F}$ and $\mathrm{G}-\mathrm{H}$, respectively, in the XTE J1701-462 sequence, and $\mathrm{K}+\mathrm{L}$ and $\mathrm{M}$ in the Cyg X-2 sequence. As the overall intensity changes and the MAXI J0556-332 tracks gradually evolve in shape, both the upper and lower vertices follow straight lines in the HID, as observed for XTE J1701-462 and Cyg X-2. The RXTE coverage of MAXI J0556-332 ended before the outburst came to an end, while the source was still showing Sco-like Z behavior. It may subsequently have passed through the atoll soft and hard states on its way back to quiescence.

Finally, we note that the atoll transient XTE J1806-246 likely entered the Sco-like Z source regime briefly at the peak of its outburst in 1998 (Wijnands \& van der Klis 1999). Observations during the outburst peak show the source tracing out a curved (partial) track resembling the lower $\mathrm{NB}+\mathrm{FB}$ part of a Sco-like $\mathrm{Z}$ track. As one would expect for a transition from the atoll to $Z$ regime, this track is at lower color values (and higher intensities) with respect to the observations in the atoll soft state taking place before and after the outburst peak. While the source traced out this track, a 7-14 Hz QPO was detected, whose frequency increases as a function of position along the track (going from the NB to FB), consistent with the behavior of the normal/flaring-branch oscillation in $\mathrm{Z}$ sources.

The three sources discussed in this section, along with the four sources analyzed in this paper, constitute the entire sample of NS-LMXBs known or believed to have shown transitions between at least two of the three main NS-LMXB subclasses (Cyg-like Z, Sco-like Z, atoll). The fact that these three additional transients show behavior seemingly largely consistent with the other four sources (e.g., the relative luminosity dependence of the subclasses) strengthens our conclusion at the end of Section 4.1. 


\subsection{Role of the Mass Accretion Rate}

The spectral fitting results of Lin et al. (2009b) indicate that the secular evolution of XTE J1701-462 was driven by changes in the mass accretion rate-they find that the $\dot{M}$ inferred from the accretion disk component in the spectra at the upper and lower vertices decreases monotonically when moving left along the two vertex lines in the HID. (See also discussion in H10.) Based on the similarities in secular behavior between XTE J1701-462 and the three sources studied in this paper we conjecture that the secular evolution of Cyg X-2, Cir X-1, and GX 13+1 is also driven by changes in the mass accretion rate. The case is clearest for Cyg X-2, whose behavior is most regular of the three sources and closest to that of XTE J1701-462. The fact that the overall intensity of the Cyg X-2 tracks decreases monotonically as they gradually evolve in shape and move along the vertex lines to higher color values makes it plausible that the mass accretion rate in Cyg X-2 decreases monotonically along the sequence, as seems to be the case for XTE J1701-462.

Although there are some important differences between the evolution of Cir X-1 and XTE J1701-462, the global properties are still very similar. The overall large intensity decrease from the Cyg-like tracks in the earlier panels to the Sco-like (and finally atoll) tracks in the later panels suggests a decreasing mass accretion rate going from panel A to L. However, the weaker correlation between track shape and location in the HID compared to the other sources - in particular manifested in the jumping back and forth in the HID seen during part of the sequence (panels $\mathrm{F}-\mathrm{I}$ ) — suggests that the mass accretion rate progression may not be strictly monotonic along the sequence. In Section 4.5 we suggest that these "discrepancies" between the track shape and location in the HID may be the result of rapid changes in the mass accretion rate.

Despite the differences in the behavior of GX $13+1$ compared to the other three sources we find it most likely that changes in the mass accretion rate are also responsible for the secular evolution of GX $13+1$ and that, as in the other sources, $\dot{M}$ decreases as the source moves up the vertex line in the $\mathrm{CD}$-i.e., going from panel $\mathrm{A}$ to $\mathrm{F}$ in Figure 12. As the tracks become overall spectrally harder the maximum intensity of a given track decreases, and the intensity swings along the track become smaller, as observed for the other three sources, although incompleteness in the tracks in panels A and B needs to be invoked to explain their deviations from this trend. Such incompleteness can, however, not explain the fact that the lower vertex in the HID is at lower intensities in those two tracks than in tracks that we suggest exhibit lower mass accretion rates, opposite to the behavior of XTE J1701-462.

Our results support the idea that the mass accretion rate is responsible for changes between subclasses in individual sources, with Sco-like Z behavior appearing at lower accretion rates and overall luminosities than Cyg-like $\mathrm{Z}$ behavior (and atoll behavior appearing at accretion rates that are lower still). However, in this picture other parameters, such as the neutron star spin, mass, and magnetic field, may still affect the luminosity scale of the subclass sequence-i.e., the range in luminosity (and mass accretion rate) over which a given subclass appears may differ between sources.

Lin et al. (2009a, 2009b) showed that for its best-estimate distance of $8.8 \mathrm{kpc}$, XTE J1701-462 reached super-Eddington luminosities during its Cyg-like stage. Even for a distance that is $30 \%$ smaller this would have been true. The super-Eddington nature of the Cyg-like tracks is further supported by observations of two Sco-like Z sources with accurately known distances, Sco X-1 (2.8 $\pm 0.3 \mathrm{kpc}$; Bradshaw et al. 1999) and LMC X-2 (50 $\pm 2 \mathrm{kpc}$; Pietrzyński et al. 2013). Spectral fits indicate that those sources are either around Eddington or mildly super-Eddington (Bradshaw et al. 2003; Agrawal \& Misra 2009). Given that Cyg-like behavior is observed at significantly higher overall intensities than Sco-like behavior in XTE J1701-462, Cyg X-2, and Cir X-1, this suggests that Cyg-like $\mathrm{Z}$ tracks may in general exhibit luminosities well above the Eddington limit.

As the sources analyzed in this paper evolve from Sco-like to Cyg-like $\mathrm{Z}$ behavior, it is interesting to see a large increase in the dynamic range (i.e., ratio of maximum to minimum intensity) of the $\mathrm{Z}$ tracks. This is most clearly seen in the tracks of XTE J1701-462, Cyg X-2, and Cir X-1, although this also holds for GX 13+1, apart from the possibly very incomplete track in panel A (Figure 12). The most extreme intensity swings are seen in Cir X-1, where we observe changes in the intensity (going between the tip of the dipping FB and the upper vertex) by factors up to 8 in periods as short as 20 minutes in the highest-intensity tracks. In these extreme Cyg-like Z tracks, Cir X-1 seems likely to have reached significantly higher luminosities (perhaps by a factor of $\sim 3$ ) and exhibited significantly higher mass accretion rates than observed in XTE J1701-462 at the peak of its outburst. Given that the large Cyg-like intensity swings observed in Cir X-1 (as well as XTE J1701-462 and Cyg X-2) occur in inferred luminosity ranges that are near- or super-Eddington, it seems likely that radiation pressure effects play an important role. While it is unlikely that the mass accretion rate toward the inner parts of the accretion flow varies this strongly on such a short timescale, strong radiation pressure may result in substantial changes in the geometry of the inner accretion flow, with a (large) fraction of the mass inflow possibly being redirected into wind or jet outflows, resulting in large intensity swings.

Given the near-/super-Eddington nature of the $\mathrm{Z}$ sources, it is also interesting to compare them with the transient ultraluminous X-ray source M82 X-2. This source was recently identified as an accreting neutron star (Bachetti et al. 2014) and has been seen to reach a maximum luminosity of $\sim 1.8 \times 10^{40} \mathrm{erg} \mathrm{s}^{-1}$ (Feng et al. 2010), which is a factor of 30 higher than the peak luminosity of XTE J1701-462 (Lin et al. 2009b). However, the short-term light curves of M82 X-2 presented in Kong et al. (2007) do not show any of the strong luminosity swings that characterize the Cyg-like Z sources. A possible explanation for this is that due to the much higher neutron star magnetic field in M82 X-2 (as inferred from the strong pulsations in the light curve; Bachetti et al. 2014) the accretion flow geometry in that source is very different from that in the Cyg-like $\mathrm{Z}$ sources, perhaps preventing the strong feedback mechanisms that we think may cause the large luminosity swings.

Three of the sources studied in this paper (XTE J1701-462, Cyg X-2, and GX 13+1) were also part of a large-sample study of NS-LMXB behavior by Muñoz-Darias et al. (2014). These authors find that the Cyg-like and Sco-like Z sources occupy a region of the rms-luminosity diagram that partly overlaps with that of the luminous black hole X-ray binary GRS $1915+105$. We note, however, that overall the Z sources and GRS 1915+105 behave very differently (e.g., Belloni et al. 2000), suggesting that 
at near/super-Eddington luminosities the observed properties of NS-LMXBs and black hole X-ray binaries are quite different, perhaps (in part) due to the increased importance of radiation feedback from the neutron star surface.

\subsection{Nonstationary Accretion in Cir X-1}

As discussed in Sections 3.3 and 4.4, the secular behavior of Cir X-1 is not as regular as that of XTE J1701-462 and Cyg X-2, as for example manifested in deviations of the track vertices from a straight line path (especially in the HID). We suspect that this may be the result of the highly nonstationary accretion in Cir X-1, thought to arise from the binary's eccentric orbit. From transient LMXBs it is known that highly nonstationary accretion can lead to substantial hysteresis (e.g., Maccarone \& Coppi 2003; Homan \& Belloni 2005), with certain source states being observed over a much larger luminosity range than would be the case for slowly changing accretion rates. Yu \& Yan (2009) showed that these effects become stronger as the changes in mass accretion rate become faster. We speculate that, analogously, highly nonstationary accretion could be a possible explanation for certain $\mathrm{Z}$ track shapes being observed over a range of luminosities, rather than at a single location along the vertex lines. While all four sources in our sample undergo nonstationary accretion, which gives rise to their secular evolution, the rate at which the mass accretion rate varies with time is likely considerably higher in Cir X-1 than in the other three sources. This is suggested by the fastest timescales on which we observe secular evolution in Cir X-1, which are shorter than in the other sources. The fastest secular evolution seen for XTE J1701-462 took place during the earliest stages of the outburst (panels $\mathrm{A}-\mathrm{C}$ ). Inspection of the SID indicates that the lower vertex of the HID track in panel $\mathrm{A}$ is at $\sim 1900$ counts $\mathrm{s}^{-1} \mathrm{PCU}^{-1}$ rather than the $\sim 1600$ counts $\mathrm{s}^{-1} \mathrm{PCU}^{-1}$ implied by the vertex line shown; we see indications for a similar, but smaller, shift in panel B. We also note that MAXI J0556-332 showed strong deviations from a straight vertex line (which it otherwise followed) during the rapid rise of its outburst (J. Homan et al. 2015, in preparation). This lends support to our hypothesis that rapid changes in the mass accretion rate underlie the observed deviations in Cir X-1.

An additional factor that may play a role in the somewhat irregular behavior of Cir X-1 is the fact that the neutron star spin, the companion spin, and the orbital axis are likely mutually misaligned in this system (Brandt \& Podsiadlowski 1995; Heinz et al. 2013). This can lead to precession of the neutron star spin axis, which may in turn affect the rotation of the inner accretion flow. Changes in the projected area of the inner accretion flow might then result in similar track shapes being observed at different X-ray fluxes.

\subsection{The Z Source Classification of $G X 13+1$}

Overall, we find that the shape and evolution of the CD/HID tracks of GX 13+1 show much stronger similarities to the other three sources analyzed in this paper than to atoll sources. Most generally, just the fact that we see gradual evolution in the shape of large-scale tracks traced out by the source as the locations of the tracks shift over a considerable range in the CD and HID points to a $\mathrm{Z}$ source, since such secular evolution in the CD/HID has never been observed in atoll sources-only in $\mathrm{Z}$ sources.
Moreover, the specifics of this evolution show many similarities to those of XTE J1701-462, Cyg X-2, and Cir X-1, as discussed in Section 3.4. Furthermore, our identification of track segments in the GX 13+1 sequence (panels D and E in Figure 12) as the $\mathrm{HB}$ and $\mathrm{HB}$ upturn (see discussion in Section 3.4.1) is supported by the detection of a highly significant $\sim 22-29 \mathrm{~Hz}$ QPO in the corresponding timing data of these segments (J. K. Fridriksson et al. 2015, in preparation). These frequencies are considerably lower than the 57-69 Hz QPO found by Homan et al. (1998) in parts of the tracks that we now classify as the upper part of the NB (see also Schnerr et al. 2003). If we interpret the QPO as an $\mathrm{HBO}$, the decrease in its frequency as the source moves onto the $\mathrm{HB}$ from the upper NB is consistent with what has been seen in other $\mathrm{Z}$ sources (see, e.g., Jonker et al. 2000 and Homan et al. 2002).

Muñoz-Darias et al. (2014) find that in their source sample there are systematic differences between the ranges in fastvariability level (broadband fractional rms) shown by the different NS-LMXB subclasses. The Cyg-like Z sources reach the highest fractional rms values (up to $\sim 10 \%-15 \%$ ), followed by the Sco-like $\mathrm{Z}$ sources (with peak values in the $\sim 5 \%-10 \%$ range), while bright persistent atoll sources and transient atolls in a bright soft state are typically observed at $\lesssim 5 \%$. MuñozDarias et al. (2014) find that the rms values oberved for XTE J1701-462 in the different subclasses are consistent with this. The highest fractional rms in the $\mathrm{Z}$ sources is observed on the $\mathrm{HB}$, and we find that on the presumed $\mathrm{HB}$ segments GX $13+1$ reaches fractional rms values as high as $\sim 9 \%-10 \%$ (J. K. Fridriksson et al. 2015, in preparation). This seems more consistent with $\mathrm{Z}$ than bright atoll behavior. We also note that the hard color range over which these GX $13+1 \mathrm{HB}$ segments are observed $(\sim 0.6-0.8)$ is consistent with HBs observed in both persistent and transient $\mathrm{Z}$ sources, but clearly too low to be consistent with the atoll hard state (Fridriksson 2011).

Considering, in addition to the above, the timescale on which the GX $13+1$ tracks are traced out (hours to $\sim 1$ day instead of the days/weeks timescales usually observed in atoll sources), the extreme scarcity of observed type I X-ray bursts from the source (Fleischman 1985; Matsuba et al. 1995; Galloway et al. 2008), and the reported radio brightness/behavior (Fender \& Hendry 2000; Homan et al. 2004) we confidently classify GX $13+1$ as a $Z$ source, one that switches between Sco- and Cyg-like $\mathrm{Z}$ behavior. However, the behavior of the source in the HID remains somewhat puzzling when compared with XTE J1701-462, Cyg X-2, and Cir X-1—especially the broad appearance of the tracks and the fact that in the spectrally harder tracks the NB/FB vertex is at higher, not lower, intensities than in the softer tracks.

This behavior may be connected to the fact that GX $13+1$ is probably viewed at a high inclination angle (perhaps $60^{\circ}-80^{\circ}$ ) as indicated by observations of absorption dips in this system on several occasions (Díaz Trigo et al. 2012; D’Aì et al. 2014; Iaria et al. 2014). It was recently shown for black hole X-ray transients that the shape of their HID tracks depends on the inclination angle (Muñoz-Darias et al. 2013). While it is not fully understood how inclination affects the shape of the HID, it is likely that an inclination dependence of the HID is present in NS-LMXBs as well, with different physical components, such as the accretion disk and boundary layer, being affected differently by changes in the viewing angle. 


\section{SUMMARY AND CONCLUSIONS}

It was recently shown that during its 2006-2007 outburst, the super-Eddington neutron star transient XTE J1701-462 evolved through all subclasses of low-magnetic-field NS-LMXBs as a result of changes in a single physical parameter: the mass accretion rate (Lin et al. 2009b; H10). The main goal of this paper is to study secular evolution in the CDs and HIDs of NS-LMXBs and, more specifically, to investigate whether evolution similar to that of XTE J1701-462 occurs in other sources. To this end we studied the evolution of the CD/HID tracks of three sources that show complicated secular behavior-Cyg X-2, Cir X-1, and GX 13+1-using all RXTE PCA data obtained during the lifetime of the mission. No comprehensive study of the $\mathrm{CD} / \mathrm{HID}$ behavior of these sources - using most or all of the RXTE data now available- has previously been performed. We created sequences of $\mathrm{CD} / \mathrm{HID}$ tracks - in many cases carefully piecing together data obtained throughout the RXTE mission to construct more complete individual tracks than otherwise available - that demonstrate the secular progression in the track morphologies and locations. In the case of $\mathrm{Cir} \mathrm{X}-1$, this analysis had to be preceded by the filtering out of data affected by intrinsic absorption, which otherwise strongly influences the appearance of the $\mathrm{CD} / \mathrm{HID}$.

We find that Cyg X-2 and Cir X-1 show strong similarities in their secular behavior to XTE J1701-462; in particular, both sources display gradual evolution between Cyg- and Sco-like $\mathrm{Z}$ tracks, with the latter occuring at lower intensities. Cir X-1 shows especially extreme versions of Cyg-like tracks at the highest observed intensities, with very large and rapid intensity swings but only small changes in spectral color. At the lowest observed intensities we also see clear transitions in the CD/HID from the atoll soft to hard state for Cir X-1. The very gradual evolution of the $\mathrm{FB}$ morphology that is observed (especially in XTE J1701-462 and Cyg X-2), from a dipping (Cyg-like) FB to a flaring Sco-like one, suggests that these two different forms of the FB are related phenomena.

Although GX 13+1 shows behavior that is in some ways peculiar-especially in the HID-we find that the source also displays similarities to XTE J1701-462, Cyg X-2, and Cir X-1, and overall we conclude that the properties of GX $13+1$ are strongly indicative of a $\mathrm{Z}$ source.

The fact that Cyg X-2 and Cir X-1 show evolution between different NS-LMXB subclasses that is similar to the evolution seen in XTE J1701-462 lends support to the suggestion of H10 that the behavior of XTE J1701-462 is representative of the entire class of NS-LMXBs. It also strengthens their conclusion that (at least within individual sources) Cyg-like $\mathrm{Z}$ behavior takes place at higher luminosities and mass accretion rates than Sco-like $\mathrm{Z}$ behavior. Based on the similarities to XTE J1701-462, we conjecture that the secular evolution of Cyg X-2, Cir X-1, and GX 13+1 is due to changes in the mass accretion rate in the systems, and overall our results support the notion that differences in mass accretion rate are the primary factor underlying the existence of the various NS-LMXB subclasses. We conclude that Cyg X-2 and Cir X-1-like XTE J1701-462-probably reach super-Eddington luminosities, with Cir X-1 likely reaching significantly higher luminosities and mass accretion rates than the other sources.
We thank the referee for constructive comments. This research has made use of data obtained from the High Energy Astrophysics Science Archive Research Center (HEASARC), provided by NASA's Goddard Space Flight Center.

\section{REFERENCES}

Agrawal, V. K., \& Misra, R. 2009, MNRAS, 398, 1352

Altamirano, D., Homan, J., Linares, M., et al. 2010, ATel, 2952

Altamirano, D., Ingram, A., van der Klis, M., et al. 2012, ApJL, 759, L20

Bachetti, M., Harrison, F. A., Walton, D. J., et al. 2014, Natur, 514, 202

Bałucińska-Church, M., Church, M. J., \& Gibiec, A. 2012, MmSAI, 83, 178

Bałucińska-Church, M., Schulz, N. S., Wilms, J., et al. 2011, A\&A, 530, A102

Belloni, T., Klein-Wolt, M., Méndez, M., van der Klis, M., \& van Paradijs, J. 2000, A\&A, 355, 271

Bradshaw, C. F., Fomalont, E. B., \& Geldzahler, B. J. 1999, ApJL, 512, L121 Bradshaw, C. F., Geldzahler, B. J., \& Fomalont, E. B. 2003, ApJ, 592, 486

Brandt, N., \& Podsiadlowski, P. 1995, MNRAS, 274, 461

Chakraborty, M., Bhattacharyya, S., \& Mukherjee, A. 2011, MNRAS, 418, 490

Church, M. J., Gibiec, A., Bałucińska-Church, M., \& Jackson, N. K. 2012, A\&A, 546, A35

Clarkson, W. I., Charles, P. A., \& Onyett, N. 2004, MNRAS, 348, 458

D’Aì, A., Bozzo, E., Papitto, A., et al. 2012, A\&A, 543, A20

D’Aì, A., Iaria, R., di Salvo, T., et al. 2014, A\&A, 564, A62

Díaz Trigo, M., Sidoli, L., Boirin, L., \& Parmar, A. N. 2012, A\&A, 543, A50

Fender, R. P., \& Hendry, M. A. 2000, MNRAS, 317, 1

Feng, H., Rao, F., \& Kaaret, P. 2010, ApJL, 710, L137

Fleischman, J. R. 1985, A\&A, 153, 106

Fridriksson, J. K. 2011, PhD thesis, Massachusetts Institute of Technology

Fridriksson, J. K., Homan, J., Wijnands, R., et al. 2010, ApJ, 714, 270

Fridriksson, J. K., Homan, J., Wijnands, R., et al. 2011, ApJ, 736, 162

Galloway, D. K., Muno, M. P., Hartman, J. M., Psaltis, D., \& Chakrabarty, D. 2008, ApJS, 179, 360

Glass, I. S. 1978, MNRAS, 183, 335

Hasinger, G., \& van der Klis, M. 1989, A\&A, 225, 79

Hasinger, G., van der Klis, M., Ebisawa, K., Dotani, T., \& Mitsuda, K. 1990, A\&A, 235, 131

Heinz, S., Sell, P., Fender, R. P., et al. 2013, ApJ, 779, 171

Homan, J., \& Belloni, T. 2005, Ap\&SS, 300, 107

Homan, J., Fridriksson, J. K., Wijnands, R., et al. 2014, ApJ, 795, 131

Homan, J., Linares, M., van den Berg, M., \& Fridriksson, J. 2011, ATel, 3650

Homan, J., van der Klis, M., Fridriksson, J. K., et al. 2010, ApJ, 719, 201

Homan, J., van der Klis, M., Jonker, P. G., et al. 2002, ApJ, 568, 878

Homan, J., van der Klis, M., Wijnands, R., et al. 2007, ApJ, 656, 420

Homan, J., van der Klis, M., Wijnands, R., Vaughan, B., \& Kuulkers, E. 1998, ApJL, 499, L41

Homan, J., Wijnands, R., Rupen, M. P., et al. 2004, A\&A, 418, 255

Iaria, R., di Salvo, T., Burderi, L., et al. 2014, A\&A, 561, A99

Jonker, P. G., \& Nelemans, G. 2004, MNRAS, 354, 355

Jonker, P. G., Nelemans, G., \& Bassa, C. G. 2007, MNRAS, 374, 999

Jonker, P. G., van der Klis, M., Homan, J., et al. 2002, MNRAS, 333, 665

Jonker, P. G., van der Klis, M., Wijnands, R., et al. 2000, ApJ, 537, 374

Jonker, P. G., Wijnands, R., van der Klis, M., et al. 1998, ApJL, 499, L191

Kaluzienski, L. J., Holt, S. S., Boldt, E. A., \& Serlemitsos, P. J. 1976, ApJL, 208, L71

Kong, A. K. H., Yang, Y. J., Hsieh, P.-Y., Mak, D. S. Y., \& Pun, C. S. J. 2007, ApJ, 671, 349

Kuulkers, E., \& van der Klis, M. 1995, A\&A, 303, 801

Kuulkers, E., van der Klis, M., Oosterbroek, T., van Paradijs, J., \& Lewin, W. H. G. 1997, MNRAS, 287, 495

Kuulkers, E., van der Klis, M., \& Vaughan, B. A. 1996, A\&A, 311, 197

Lin, D., Altamirano, D., Homan, J., et al. 2009a, ApJ, 699, 60

Lin, D., Remillard, R. A., \& Homan, J. 2009b, ApJ, 696, 1257

Lin, D., Remillard, R. A., Homan, J., \& Barret, D. 2012, ApJ, 756, 34

Linares, M., Chakrabarty, D., \& van der Klis, M. 2011, ApJL, 733, L17

Linares, M., Watts, A., Altamirano, D., et al. 2010, ApJL, 719, L84

Maccarone, T. J., \& Coppi, P. S. 2003, MNRAS, 338, 189

Matsuba, E., Dotani, T., Mitsuda, K., et al. 1995, PASJ, 47, 575

Migliari, S., \& Fender, R. P. 2006, MNRAS, 366, 79

Moneti, A. 1992, A\&A, 260, L7

Muñoz-Darias, T., Coriat, M., Plant, D. S., et al. 2013, MNRAS, 432, 1330

Muñoz-Darias, T., Fender, R. P., Motta, S. E., \& Belloni, T. M. 2014, MNRAS, 443, 3270 
Murdin, P., Jauncey, D. L., Lerche, I., et al. 1980, A\&A, 87, 292

Oosterbroek, T., van der Klis, M., Kuulkers, E., van Paradijs, J., \& Lewin, W. H. G. 1995, A\&A, 297, 141

Parkinson, P. M. S., Tournear, D. M., Bloom, E. D., et al. 2003, ApJ, 595, 333

Pietrzyński, G., Graczyk, D., Gieren, W., et al. 2013, Natur, 495, 76

Psaltis, D., Lamb, F. K., \& Miller, G. S. 1995, ApJL, 454, L137

Remillard, R. A., \& Lin, D. 2006, ATel, 696

Remillard, R. A., Lin, D., Cooper, R. L., \& Narayan, R. 2006, ApJ, 646, 407

Schnerr, R. S., Reerink, T., van der Klis, M., et al. 2003, A\&A, 406, 221

Sell, P. H., Heinz, S., Calvelo, D. E., et al. 2010, ApJL, 719, L194

Shirey, R. E., Bradt, H. V., \& Levine, A. M. 1999, ApJ, 517, 472

Shirey, R. E., Bradt, H. V., Levine, A. M., \& Morgan, E. H. 1996, ApJL, 469, L21

Shirey, R. E., Bradt, H. V., Levine, A. M., \& Morgan, E. H. 1998, ApJ, 506,374

Shirey, R. E., Levine, A. M., \& Bradt, H. V. 1999b, ApJ, 524, 1048
Sugizaki, M., Yamaoka, K., Matsuoka, M., et al. 2013, PASJ, 65, 58

Tauris, T. M., Fender, R. P., van den Heuvel, E. P. J., Johnston, H. M., \& Wu, K. 1999, MNRAS, 310, 1165

Tennant, A. F., Fabian, A. C., \& Shafer, R. A. 1986a, MNRAS, 221, 27P

Tennant, A. F., Fabian, A. C., \& Shafer, R. A. 1986b, MNRAS, 219, 871

van der Klis, M. 2001, ApJ, 561, 943

van der Klis, M. 2006, in Compact Stellar X-Ray Sources, ed. W. H. G. Lewin \& M. van der Klis (Cambridge, UK: Cambridge Univ. Press), 39

Whelan, J. A. J., Mayo, S. K., Wickramasinghe, D. T., et al. 1977, MNRAS, 181,259

Wijnands, R., \& van der Klis, M. 1999, ApJ, 522, 965

Wijnands, R. A. D., van der Klis, M., Kuulkers, E., Asai, K., \& Hasinger, G. 1997, A\&A, 323, 399

Wilson-Hodge, C. A., Cherry, M. L., Case, G. L., et al. 2011, ApJL, 727, L40

Yu, W., \& Yan, Z. 2009, ApJ, 701, 1940 